INRA Prod. Anim., 2017, 30 (4), 407-422

\title{
Les bouquets de services, un concept clé pour raisonner l'avenir des territoires d'élevage
}

\author{
B. DUMONT', J. RYSCHAWY', M. DURU' ${ }^{2}$, M. BENOIT ${ }^{1}, L . D E L A B Y^{3}$,

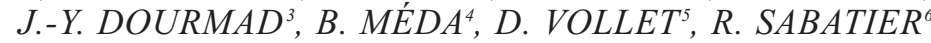 \\ ${ }^{1}$ Université Clermont Auvergne, INRA, VetAgro Sup, UMR Herbivores, 63122, Saint-Genès-Champanelle, France \\ 2 UMR AGIR, INRA, Université de Toulouse, INPT, 31326, Castanet-Tolosan, France \\ ${ }_{3}^{3}$ PEGASE, Agrocampus Ouest, INRA, 35590, Saint-Gilles, France \\ ${ }_{4}^{4}$ URA, INRA, 37380, Nouzilly, France \\ ${ }^{5}$ Université Clermont Auvergne, AgroParisTech, INRA, Irstea, VetAgro Sup, UMR Territoires, \\ 63000, Clermont-Ferrand, France \\ - INRA, AgroParisTech, Université Paris-Saclay, UMR SAD-APT, 75000, Paris, France \\ Courriel : bertrand.dumont@inra.fr
}

Les interrelations entre les impacts et les services rendus par l'élevage impliquent de ne pas considérer ces effets indépendamment les uns des autres. Dans cet article, nous analysons les approches qui permettent de décrire et d'analyser les bouquets de services fournis par l'élevage dans différents types de territoires. Nous proposons des pistes de recherche à approfondir afin de mieux valoriser ces différents services.

L'élevage représente une activité majeure pour l'économie de nombreux territoires européens et a des impacts qui varient fortement d'un territoire à l'autre. Au-delà des controverses sur les conséquences environnementales de l'élevage (FAO 2006, Rockström et al 2009), et sur les conditions de vie et d'abattage des animaux (Larue 2015), l'élevage rend différents services à la société qu'il convient de révéler, et si possible de quantifier. Le concept de multifonctionnalité a mis en avant qu'audelà de sa finalité productive, l'élevage avait de nombreux autres effets « secondaires » ou externalités ${ }^{1}$. Le concept de multifonctionnalité semble aujourd'hui supplanté par les approches en termes de services qui recouvrent à la fois les services écosystémiques (d'approvisionnement, de régulation et culturels) et les externalités positives de l'agriculture ; il reste toutefois utilisé y compris dans le domaine de l'élevage (Brummel et Nelson 2014).

Définis à l'interface entre écologie et économie, les services écosystémiques concernent les bénéfices fournis à la société par les écosystèmes naturels (MEA 2005). Les services écosystémiques sont alors définis comme la résul- tante de l'attribution par des bénéficiaires (i.e. le socio-système) d'une valeur positive à certaines fonctions ou structures écosystémiques. La biodiversité rend aussi des services à l'agriculture (appelés « services intrants ») tels que la stabilité des rendements fourragers, la qualité des sols ou la pollinisation. La notion de services écosystémiques, initialement largement dévolue aux écosystèmes naturels, a depuis été étendue aux agroécosystèmes qui constituent une large part des écosystèmes continentaux. Bien que de plus en plus reconnu, le rôle de l'élevage dans la gestion des services écosystémiques est encore mal évalué, audelà de l'effet des modes de conduites des parcelles sur la biodiversité (Sabatier et al 2014, Milberg et al 2016, Ravetto Enri et al 2017), et des conséquences économiques de la gestion de paysages par l'élevage (par exemple le prix de location des gîtes ruraux, Mollard et al 2006). La contribution de l'élevage au bien-être de la société est cependant mis en avant par les systèmes herbagers qui entretiennent des espaces à forte valeur patrimoniale (Lemauviel-Lavenant et Sabatier 2017, Vollet et al 2017, ce numéro) et par ceux, encore minoritaires, qui misent sur une image de qualité et un lien renforcé entre le producteur et le consommateur (Benoit et Méda 2017, Delfosse et al 2017, Vollet et al 2017, ce numéro). Reconnaître les services fournis par l'élevage à la société n'occulte pas la nécessité d'examiner conjointement ses effets négatifs (le terme d'impacts connoté négativement est alors utilisé). On trouve ainsi dans la littérature la notion de «dys-services », qui correspond à des nuisances pour l'homme, issues soit du fonctionnement de l'écosystème (Lele et al 2013), soit des externalités négatives de l'élevage (pollutions et dégradations) qui ne sont pas prises en compte dans les services écosystémiques listés par le MEA.

Rodriguez et al (2006), Bennett et al (2009) et différents auteurs après eux, ont pointé la nécessité de ne pas considérer les services écosystémiques indépendamment les uns des autres du fait des interactions entre les processus écologiques qui les sous-tendent. Le cadre des « bouquets de services » permet de considérer la coexistence d'effets positifs et négatifs des activités qui structurent un territoire à un instant donné, et les interrelations entre services (RaudseppHearne et al 2010). Le concept de «système socio-écologique » permet d'examiner les interactions entre un système

1 Terme utilisé par les économistes pour désigner toutes situations où l'activité de production ou de consommation d'un agent exerce une influence sur le bienêtre d'un autre agent, sans que cette interaction ne fasse l'objet d'une transaction économique (Picard 1994). 
social composé d'acteurs, individuels et collectifs, mobilisant des technologies et des infrastructures pour gérer des ressources et un système écologique générant ces ressources (Berkes et al 2003, Anderies et al 2004). L'élevage peut être vu comme un système socio-écologique (Duru et al 2017, ce numéro) au sein duquel l'inventaire des couplages entre services renvoie aux notions de synergies, d'antagonismes et de compromis (l'encadré 1 précise l'usage de ces termes). Dans le cas fréquent où l'augmentation d'un service est contrebalancée par la diminution d'un autre service, il s'agit de rechercher un compromis dont le résultat peut être jugé différemment selon les acteurs. La nécessaire prise en compte des préférences des acteurs fait émerger l'importance d'une approche socio-technico-écologique (Lescourret et al 2015). Les compromis sont alors vus sous l'angle des négociations sociales et des délibérations politiques à la recherche d'un bien commun territorialisé. Une telle démarche est engagée dans les territoires d'élevage herbagers (Rodríguez-Ortega et al 2014) et les territoires de polycultureélevage (Moraine et al 2016).

Les « service d'approvisionnement» correspondent à l'activité productive des systèmes agricoles. Celle-ci comprend la part de la production fournie par les services intrants et celle provenant d'intrants exogènes d'origine anthropique ; le service d'approvisionnement ne peut donc pas être considéré comme un service écosystémique au sens strict. Ryschawy et al (2015) ont décliné le concept de bouquets de services aux territoires d'élevage métropolitains, en considérant les services d'approvisionnements, conjointement aux services environnementaux, économiques et sociaux. Leur approche intégrait les services écosystémiques de qualité environnementale et culturels mais aussi des services dits de vitalité territoriale (emploi, maintien d'un tissu rural), essentiels lorsqu'on s'intéresse à l'avenir de l'élevage. Le cadre conceptuel dit « de la grange », élaboré à l'occasion de l'expertise scientifique collective sur les rôles, impacts et services issus des élevages en Europe (Dumont et al 2017) et détaillé dans ce numéro spécial par Duru et al (2017), permet de représenter les flux et les pressions propres ou extérieures à chaque territoire d'élevage qui s'exercent sur celui-ci. À l'instar du travail de Ryschawy et al (2015), les granges visualisent les impacts et services rendus par l'élevage dans un territoire, au-delà des seuls services écosystémiques.

Dans la première partie de cet article, nous analysons comment les cartographies de services, à des échelles allant de la petite région agricole à l'Europe entière, mettent en lumière des antagonismes et des synergies entre les princi-

Encadré 1. Usage des termes antagonismes, synergies et compromis entre services.

L'usage des termes antagonismes, synergies et compromis renvoie à deux grands ensembles de travaux : i) ceux où les relations entre services, principalement écosystémiques, sont analysées sous l'angle des interactions biophysiques (on traite alors des antagonismes et des synergies), et ii) ceux où les services sont abordés sous l'angle des préférences des acteurs dans un cadre de contraintes ou d'objectifs de politiques publiques (on recherche alors des compromis).

Lorsque la fourniture d'un service est pénalisée par celle d'un autre service, voire engendre des externalités négatives, on parle d'antagonisme. Dans le cas inverse, lorsque la fourniture d'un service est favorisée par celle d'un autre service, on parle de synergie. Dans la littérature, les termes antagonismes et synergies entre services sont utilisés pour parler i) des interactions écologiques et/ou biophysiques entre services (Bennett et al 2009, Rodríguez-Ortega et al 2014), ii) de la variabilité temporelle dans la fourniture d'un service (Kragt et Robertson 2014) et iii) de relations positives ou négatives entre services non fonctionnellement liés, telles que leur concomitance spatiale (Maes et al 2012b, Jopke et al 2015, Kirchner et al 2015).

La question des compromis est abordée soit au travers du décalage entre la fourniture d'un service et la demande (Kroll et al 2012), soit de l'évaluation contrastée des services qu'ont les différents acteurs d'un territoire (Groot et al 2010, Howe et al 2014). Les compromis sont alors le résultat d'arbitrages plus ou moins explicites dans lesquels deux parties (ou plus) qui ont des intérêts divergents font des concessions mutuelles dans le but de parvenir à un accord. Dans d'autres cas, les arbitrages résultent d'une décision «top-down » de politique publique.

paux types de services. Nous présentons ensuite trois granges synthétiques qui illustrent les bouquets de services « moyens », dans les territoires à haute densité animale (Dourmad et al 2017), les territoires herbagers (Delaby et al 2017 ; Lemauviel-Lavenant et Sabatier 2017 ; Vollet et al 2017) et les territoires de polyculture-élevage (Ryschawy et al 2017). Ces granges traduisent la situation actuelle (ce qui est) à un grain qui révèle avant tout l'effet des systèmes dominants dans le territoire. Nous prolongeons cette analyse à des échelles plus fines (bassin versant, canton, exploitation) pour montrer qu'il est possible de dépasser certains antagonismes entre services en prenant en compte les temporalités (par exemple les périodes de pâturage), l'hétérogénéité spatiale, et les interactions techniques ou organisationnelles entre les différents systèmes d'élevage (et de cultures) qui coexistent dans un même territoire. Ceci nous conduit à identifier des voies d'amélioration pour chaque type de territoire (ce qui pourrait être). La dernière partie de cet article traite de scénarios prospectifs réalisés à une échelle nationale, européenne ou globale. L'intérêt de ces scénarios est d'explorer les conséquences du changement climatique, et d'évolutions plus tranchées des modes de production et de consommation, en particulier une forte réduction de la part des protéines animales dans l'alimentation des européens. Ces différentes " clefs de lecture » permettent d'appréhender l'ensemble des services rendus par l'élevage, et qui sont souvent ignorés par le marché et les citoyens. Elles les mettent au regard de ses impacts négatifs sur l'environnement et la santé humaine, et donnent ainsi à voir comment rechercher des compromis pour aboutir à des bouquets de services plus « équilibrés ». Nous concluons en proposant des pistes de recherches à approfondir afin de mieux valoriser la diversité des services fournis par l'élevage.

\section{1 / Apports et limites des cartographies de services}

\section{1 / Les cartographies de services distinguent les territoires pro- ductifs de territoires qui rendent davantage de services culturels et de régulation}

Les cartographies à l'échelle d'un pays mettent en évidence des corrélations entre la présence de l'élevage dans certaines parties du territoire et la fourniture de tel ou tel bouquet de services. De manière récurrente, on observe une corrélation négative entre le service d'approvisionnement d'une part, et les services culturels et de régulation d'autre part. En France métropolitaine, Ryschawy et al (2015) ont identifié quatre types de territoires d'élevage selon les bouquets de services qu'ils fournissent. Le premier, en Bretagne et Pays-de-Loire, correspond à une forte expression des services d'approvisionnement et de vitalité rurale, et à une moindre expression des services contribuant à la qualité environnemen- 
Figure 1. Distribution spatiale de certains services d'approvisionnement (productions animales et grandes cultures), culturels (usage pour la chasse) ou de régulation (du climat via la séquestration du carbone) au Danemark (adapté de Turner et al 2014). L'analyse conjointe de 11 services écosystémiques (dont les quatre représentés ici) met en évidence une spécialisation territoriale autour de l'agriculture (en vert sur la carte de droite) ou de la forêt (bleu marine), mais aussi des zones intermédiaires (bleu ciel) et des territoires plus multifonctionnels (orange) par exemple dans les espaces péri-urbains.
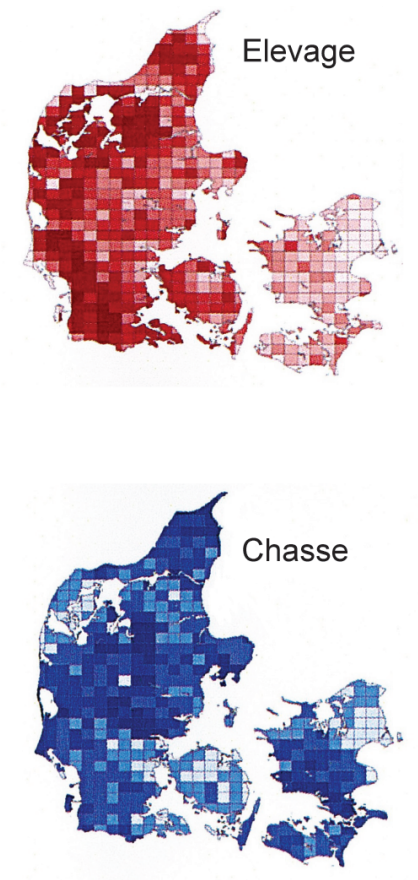

tale. Le deuxième type, dans le Massif central et en Basse Normandie, correspond à une forte expression des services de qualité environnementale et de certains services culturels (paysages patrimoniaux, produits de qualité) associés à moins de services d'approvisionnement et de vitalité rurale (excepté la contribution de l'élevage à l'emploi local). Le troisième type, dans les Alpes, le Jura et les Pyrénées, correspond à une forte expression des services de qualité environnementale et de patrimoine associés à un faible niveau des services d'approvisionnement et de vitalité rurale. Enfin, le quatrième type, en bordure des bassins céréaliers, correspond à des niveaux de services rendus par l'élevage plus faibles en raison de l'abandon progressif de l'élevage au profit des cultures. Cette typologie a depuis été étendue à l'échelle de l'Europe, en regroupant le deuxième et le troisième type au sein d'un type herbager qui se décline le long d'un gradient de densités animales (Hercule et al 2017, ce numéro).

D'autres travaux permettent de décrire et de cartographier les bouquets de services fournis par l'activité agricole, sans toujours distinguer les effets propres à l'élevage. En Autriche, Kirchner et al (2015) montrent une ségrégation spatiale entre des zones dédiées à la production (grandes cultures, fourrages et biomasse énergétique ici considérés de manière conjointe) qui contribuent fortement au produit national brut, et d'autres propices à la préservation de la biodiversité. Ces dernières sont caractérisées par leur
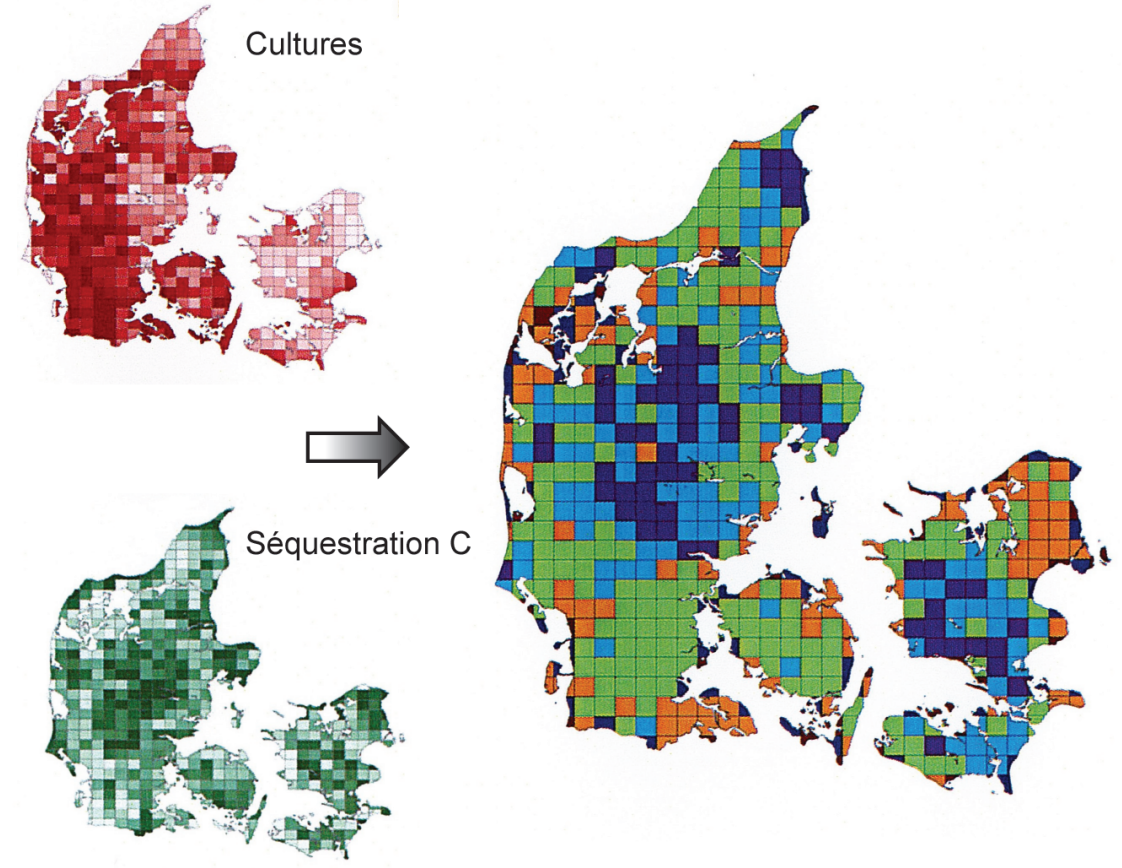

« naturalité » et contribuent à la régulation du climat puisque des pratiques telles que la réduction de la fertilisation minérale ont un effet positif conjoint sur la biodiversité et les émissions de Gaz à Effet de Serre (GES). Le revenu des exploitations est plus élevée dans le second type en raison des subventions perçues pour la mise en œuvre de mesures agri-environnementales.

Au Danemark, la localisation de l'élevage et des grandes cultures est très corrélée (Turner et al 2014) sans que, pour l'élevage, on puisse distinguer la contribution relative des vaches laitières et des porcs. Une telle combinaison répond au besoin de terres pour épandre les déjections animales et satisfaire aux exigences de la directive « Nitrates ». L'étude met en évidence une spécialisation des territoires autour de l'agriculture d'une part, et de la forêt d'autre part avec un bouquet de services écosystémiques dans lequel prévalent la régulation du climat et la chasse (figure 1). Au-delà de ces spécialisations territoriales marquées, il apparaît des territoires multifonctionnels dans lesquels les bouquets de services sont plus « équilibrés » (figure 1). C'est le cas de certaines zones herbagères (Dick et al 2011, Ryschawy et al 2015) et des espaces péri-urbains (van Oudenhoven et al 2012, Turner et al 2014), où l'élevage, au-delà de sa fonction productive, répond aux attentes des citoyens en termes de loisirs (centres équestres, entretien d'espaces attractifs à proximité immédiate des villes, Delfosse et al 2017) et rend différents services de régulation (captation des particules fines et limitation des risques de crues par les prairies).

\section{2 / Des tendances qui se retrouvent à l'échelle du continent européen}

À l'échelle de l'Europe entière, Maes et al (2012b) ont proposé une carte des principaux services écosystémiques et de la biodiversité avec une résolution de $10 \mathrm{~km}$. La biodiversité y est appréciée par trois indicateurs : la part de richesse spécifique globale qui subsiste dans le milieu relativement au même milieu non impacté par l'homme (Alkemade et al 2009), la part du territoire occupée par les zones Natura 2000, et un indice de diversité des arbres. Le long d'un gradient de biodiversité croissant, on constate une augmentation de la fourniture de services écosystémiques jusqu'à un certain seuil, au-delà duquel celle-ci reste stable. Pour Maes et al (2012b), les territoires d'élevage fournissent peu de services de régulation et de services culturels comparés à la forêt ou aux zones humides, avec toutefois une plus forte probabilité de concilier production animale et conservation de la biodiversité lorsque les densités animales décroissent. Lorsque les animaux élevés en bâtiments (dont l'alimentation n'est pas produite dans le territoire) ne sont pas pris en compte dans ces analyses, on ne retrouve plus de corrélation négative entre les densités animales et la fourniture de services de régulation ou l'usage récréatif des espaces (Jopke et al 2015). 


\section{3 / Intérêts et limites des approches cartographiques}

Les cartographies de services permettent de distinguer les territoires productifs de territoires ayant une plus forte valeur environnementale à des échelles allant de la petite région agricole (Palomo et al 2014, Van der Biest et al 2014) à l'Europe entière (Maes et al 2012b). Elles offrent ainsi une base pour proposer des politiques d'aménagement ciblées en donnant à voir l'intensité et la localisation des compromis à résoudre selon les territoires. Dans ces démarches, les aspects socioculturels ne sont pas systématiquement pris en compte, en raison d'un manque d'indicateurs génériques pour quantifier les services culturels et les emplois créés par l'élevage.

Malgré l'intérêt de ces travaux, trois limites doivent être rappelées. La première concerne le type d'indicateurs retenus pour évaluer les services. Dans de nombreuses études, le choix des indicateurs et l'échelle d'analyse sont conditionnés par la disponibilité d'indicateurs quantitatifs dans des bases de données nationales ou européennes (RaudseppHearne et al 2010, Ryschawy et al 2015), ou par la possibilité de les simuler par modélisation. Ces choix méthodologiques ont une influence majeure sur les prédictions que ces modèles permettent de réaliser, comme l'illustre les conclusions du travail de Jopke et al (2015).

La deuxième limite repose sur le fait que la spatialisation des bouquets de services correspond en général à une projection statique de l'effet des systèmes dominants sur une carte. Les analyses aux échelles larges gomment ainsi la diversité des systèmes d'élevage qui coexistent dans les territoires, et masquent les contributions positives que peuvent avoir certains systèmes minoritaires. Ainsi, la région Pays-de-la-Loire apparaît comme un territoire à haute densité animale (Ryschawy et al 2015, Hercule et al 2017) dont le bouquet de services est décrit figure 2 , alors qu'on y retrouve également $40 \%$ de la production de volailles Label Rouge française qui génère un bouquet de services très différent (Benoit et Méda 2017).

Enfin, la concomitance spatiale de différents services écosystémiques est en général considérée comme une relation de cause à effet, alors qu'elle ne repose souvent que sur de simples corrélations entre les indicateurs retenus pour évaluer les services (Hamann et al 2015). Les interrelations entre services ne devraient donc être analysées comme des liens de cause à effets, que si des relations directes entre services ont été explicitées ou les leviers déterminant plusieurs services précisés (Bennett et al 2009). Audelà de la projection des bouquets de services sur une carte, nous recommandons donc de réaliser des analyses d'autocorrélations spatiales, qui seules permettent de révéler les processus spatiaux sous-jacents à la fourniture des services (Turner et al 2014).

\section{2 / Comprendre les compro- mis entre services à l'échelle des territoires et des systèmes}

Complémentaires de ces cartographies, d'autres travaux analysent les bouquets de services à l'échelle plus fine des systèmes ou des territoires. Les services écosystémiques y ont jusqu'ici surtout été considérés individuellement ou par deux afin de mettre en évidence des synergies et des antagonismes, et les approches par bouquets de services restent minoritaires (Tancoigne et al 2014). Le cadre conceptuel de la grange (Duru et al 2017), permet toutefois de représenter les flux et les pressions propres ou extérieures à chaque territoire, et l'ensemble des impacts et services rendus par l'élevage audelà des seuls services écosystémiques. Nous l'utilisons ici pour visualiser les principaux compromis à rechercher dans les territoires à haute densité animale, les territoires herbagers et les territoires de polyculture-élevage. Différents approfondissements prennent en compte les complémentarités techniques et organisationnelles entre les systèmes qui coexistent dans un territoire. La recherche de compromis mobilise par ailleurs des connaissances sur les processus biologiques qui sous-tendent les services. Ils sont ici synthétisés pour chacun des trois territoires précités.

\section{1 / Bouquets de services et compromis dans les territoires à haute densité animale}

Ces territoires d'élevage se sont développés dans une logique de maximisation des quantités produites par unité de surface et par animal, en misant sur une forte concentration territoriale, une spécialisation des ateliers, et une augmentation de la productivité du travail (Roguet et al 2015). Avec 29\% du cheptel européen (UGB) sur seulement $10 \%$ de la SAU (Hercule et al 2017), le bouquet de services est très déséquilibré en faveur du service d'approvisionnement (avec des produits bon marché accessibles au plus grand nombre) et de l'emploi, mais peu de services de qualité environnementale (figure 2, tableau 1, voir Dourmad et al
2017 pour une illustration de la variabilité autour de ce bouquet de services moyen). Du fait des dommages environnementaux occasionnés par ces formes d'élevage, une priorité des politiques publiques est la recherche de compromis entre les objectifs de production et la limitation des pollutions et des nuisances. L'élevage dit « industriel » est également remis en cause en raison de ses conséquences sur les conditions de vie et d'abattage des animaux. Les conditions de travail des éleveurs et des salariés des industries agroalimentaires sont difficiles dans ces formes d'élevage, dont l'acceptabilité locale s'explique par les nombreux emplois créés.

Les excédents d'azote en Bretagne, à l'origine de nuisances perceptibles par les citoyens (odeurs, nitrates, algues vertes), illustrent les conséquences d'un déséquilibre territorial entre une forte charge animale et un milieu particulièrement sensible. L'élevage a dans ce cas non seulement un impact local lié aux limites de la capacité de valorisation des effluents, mais aussi un impact délocalisé de par les importations de soja et de céréales pour nourrir le bétail (Chaudhary et Kastner 2016). Les arbitrages locaux ont été réalisés par la voie réglementaire, en particulier la directive « Nitrates » et le placement de la région en zone d'excédents structurels. Ces politiques publiques ont fait évoluer les pratiques d'alimentation des animaux, et les pratiques d'épandage, de traitement et de valorisation de leurs déjections, par exemple sous forme de fertilisants organiques commercialisés auprès des maraîchers. Une réduction du cheptel de volailles, des bovins et plus récemment des porcs a toutefois été nécessaire pour réduire la quantité d'azote organique et améliorer la qualité des eaux (Dourmad et al 2017).

Les émissions de GES sont importantes à l'échelle du territoire mais restent relativement modérées lorsqu'on les ramène au kg produit. Pour les monogastriques, c'est le résultat d'une optimisation de l'indice de conversion alimentaire des animaux, par l'alimentation et le progrès génétique (Patience et al 2015), associée à une bonne gestion des effluents dans des bâtiments plus économes en énergie (Dourmad et al 2014, Loyon et al 2016). Pour les ruminants, les émissions de méthane entérique sont diluées par les volumes de production lorsque la productivité par animal augmente, puis se stabilisent en particulier pour la production laitière (Gerber et al 2011). Ainsi, une comparaison entre des fermes bretonnes en système conventionnel et de fermes plus herbagères et moins intensives du réseau des CIVAM $^{2}$ 
Figure 2. Bouquet de services correspondant aux territoires à haute densité animale.

Comme décrit par Duru et al (2017), le pentagone central représente un territoire d'élevage avec ses éléments paysagers (haies) et les activités agro-industrielles sur lesquelles s'appuie l'élevage. Celui-ci est décrit par ses caractéristiques : espèces et densité animales, modes d'alimentation (animal vert : pâturage et fourrages, ocre : aliments concentrés dont l'ensilage de maïs). Le mode d'usage des terres est représenté par un parcellaire à deux nuances de vert pour symboliser la diversité des prairies permanentes et la présence de temporaires, et à deux nuances de jaune pour représenter la diversité des rotations culturales. Au sein du territoire, les systèmes d'élevage interagissent avec cinq interfaces. Les pictogrammes explicités dans le tableau joint symbolisent les éléments concernés par ces interfaces. La nature et l'ampleur des effets sont représentées par une flèche sortante plus ou moins large et dont la couleur indique que ceux-ci sont positifs (flèche verte), négatifs (rouge) ou mitigés (hachures) ; dans ce cas, l'effet dominant borde la flèche. Sur l'interface travail et emploi, nous distinguons les emplois directs en élevage des emplois indirects (IAA, tourisme, flèche vers personnage blanc). Les flèches entrantes indiquent l'utilisation d'intrants exogènes (qui symbolisent les effets délocalisés de l'élevage), des pressions liées aux marchés, ou (sur l'interface environnement et climat) que l'élevage bénéficie de services intrants (flèche verte) ou subit la pression de facteurs environnementaux (prédation, sécheresse... ; flèche rouge).

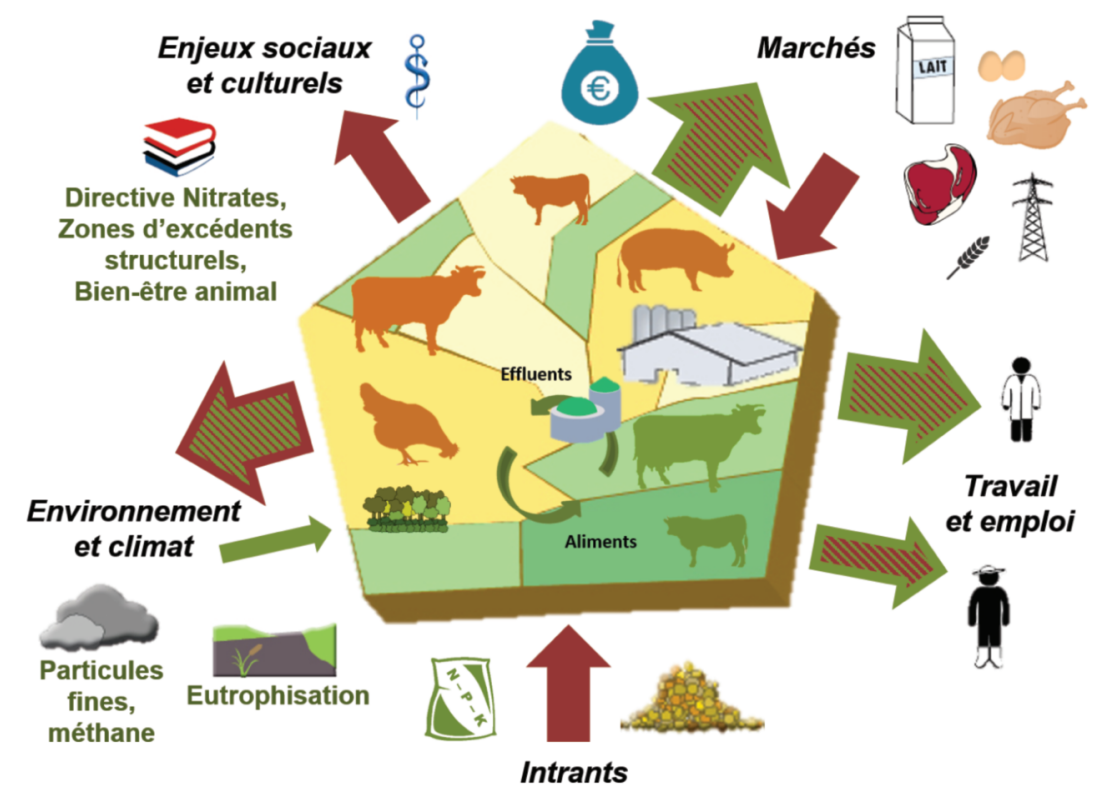

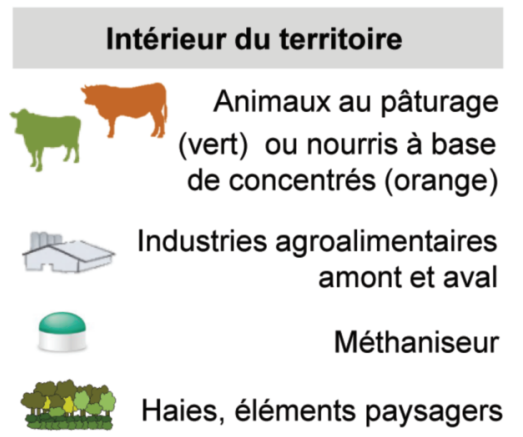

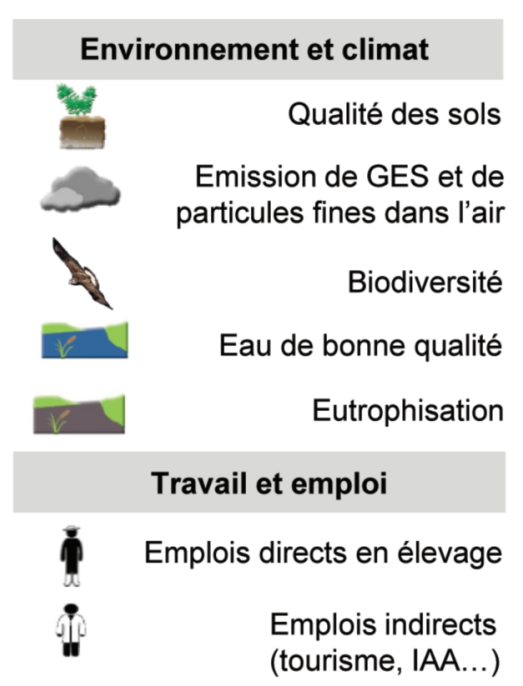

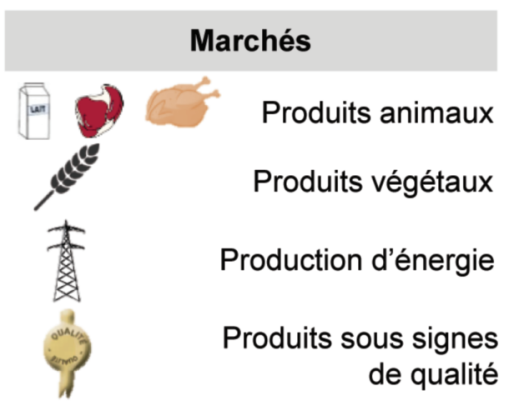

Création de valeur monétaire

Intrants

\section{Enjeux sociaux et culturels}

$\$$

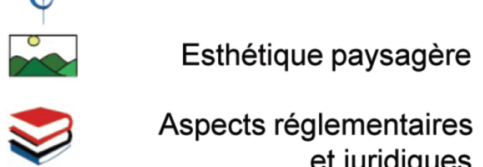

et juridiques

Patrimoine gastronomique 
Tableau 1. Atouts et impacts associés aux territoires à haute densité animale.

\begin{tabular}{|c|c|c|}
\hline Interfaces étudiées & Effets positifs & Effets négatifs \\
\hline Intrants & $\begin{array}{l}\text { - Recyclage effluents organiques } \\
\text { comme fertilisants } \\
\text { - Production d'énergie, méthanisation }\end{array}$ & $\begin{array}{l}\text { - Effets délocalisés (usage des terres, } \\
\text { pesticides, biodiversité) de la production } \\
\text { de concentrés } \\
\text { - Fertilisation minérale, pesticides }\end{array}$ \\
\hline Travail et emploi & $\begin{array}{l}\text { - Nombreux emplois dans les filières } \\
\text { agro-alimentaires et en amont } \\
\text { de la production } \\
\text { - Nombreux emplois directs } \\
\text { en élevage }\end{array}$ & $\begin{array}{l}\text { - Conditions de travail dans les abattoirs } \\
\text { et l'agro-alimentaire } \\
\text { - Pression du contexte économique et liée } \\
\text { à la perception de l'élevage « industriel » } \\
\text { par la société }\end{array}$ \\
\hline Marchés & $\begin{array}{l}\text { - Production de masse à prix } \\
\text { compétitifs } \\
\text { - Exportations massives vers les pays } \\
\text { de l'UE et les pays tiers }\end{array}$ & $\begin{array}{l}\text { - Dépendance de la conjoncture } \\
\text { internationale pour le prix des matières } \\
\text { premières et de mise en marché }\end{array}$ \\
\hline $\begin{array}{l}\text { Enjeux sociaux } \\
\text { et culturels }\end{array}$ & $\begin{array}{l}\text { - Acceptation locale liée aux emplois } \\
\text { créés }\end{array}$ & $\begin{array}{l}\text { - Atteintes au bien-être animal } \\
\text { - Pollutions de l'eau et de l'air } \\
\text { - Nuisances olfactives } \\
\text { - Antibiorésistance } \\
\text { - Risques pour la santé du fait des densités } \\
\text { animales élevées }\end{array}$ \\
\hline $\begin{array}{l}\text { Environnement } \\
\text { et climat }\end{array}$ & $\begin{array}{l}\text { - Faibles émissions de GES par kg } \\
\text { de produit (productivité, efficacité } \\
\text { alimentaire) } \\
\text { - Services intrants fournis par les } \\
\text { prairies (légumineuses) }\end{array}$ & $\begin{array}{l}\text { - Excédents d'azote } \\
\text { - Pollution des eaux de surface et des nappes } \\
\text { - Pollution de l'air ( } \mathrm{NH}_{3} \text {, particules fines). } \\
\text { - Retournement des prairies }\end{array}$ \\
\hline
\end{tabular}

révèle des émissions de GES par litre de lait similaires entre les deux systèmes; le bilan net est cependant meilleur dans les fermes CIVAM grâce à la séquestration du carbone par les prairies permanentes et les haies (Duru et Therond 2015). Le choix de l'unité fonctionnelle (e.g. selon que les émissions de GES sont ramenées au $\mathrm{kg}$ de produit, à l'hectare ou à la valeur ajoutée ; Benoit et Méda 2017 ; Salou et al 2017) et des frontières du système (Jopke et al 2015, Chaudhary et Kastner 2016) modifie également les classements entre systèmes. Logiquement, le choix de l'unité fonctionnelle devrait refléter ce qui est jugé comme étant le plus limitant : l'utilisation des terres, les volumes produits, la consommation d'eau ou d'énergie non renouvelable (Dumont et al 2014). Au-delà de ces considérations méthodologiques, l'exemple breton illustre qu'un même type de système peut être efficace par rapport à ses impacts globaux (émissions de GES), mais bien moins pour ses impacts locaux (nitrates, particules fines ; Dourmad et al 2017).

Enfin, les préoccupations relatives au bien-être animal se sont traduites par des réglementations qui ont eu pour conséquence d'améliorer conjointement les performances individuelles des animaux
(Coignard et al 2013), leur santé (Bertoni et al 2016) et par voie de conséquence l'efficience des systèmes de production. Ces réglementations diminuent le recours aux intrants médicamenteux et donc les charges opérationnelles de l'exploitation, et ont des effets positifs sur l'environnement (moins de résidus) et la santé humaine (antibiorésistance ; Marshall et Levy 2011). La surface de bâtiments allouée aux animaux est accrue ce qui réduit les volumes produits par unité de surface et génère des coûts de structure plus importants pour un même volume de production.

\section{2 / Bouquets de services et compromis dans les territoires herbagers}

Les territoires d'élevage herbagers sont essentiellement dévolus à l'élevage de ruminants. Ces systèmes se caractérisent par la recherche d'une autonomie alimentaire élevée, avec une logique d'adaptation des pratiques aux potentialités du milieu plutôt que de réduction des facteurs limitants par le recours aux intrants exogènes (Delaby et Fiorelli 2014). Ils valorisent les services intrants grâce à un recours privilégié à la prairie, sans pour autant exclure l'achat d'aliments concentrés, notamment en zones de mon- tagne où ceux-ci ne peuvent pas être produits sur l'exploitation. Les services d'approvisionnement y sont moins développés que dans les territoires à haute densité animale. En revanche, la forte expression des services de qualité environnementale et culturels leur confère une bonne image auprès du consommateur (figure 3, tableau 2, voir Delaby et al 2017 ; Lemauviel-Lavenant et Sabatier 2017 ; Vollet et al 2017 pour une illustration de la variabilité autour du bouquet de services moyen). Les services écosystémiques de qualité environnementale et culturels les plus fréquemment quantifiés sont la préservation de la biodiversité, la valeur esthétique des paysages, et la régulation du climat au travers de la séquestration du carbone par les prairies (Rodríguez-Ortega et al 2014).

Les conditions de production de ces systèmes (en particulier des durées d'élevage plus longues) génèrent plus d'émissions de GES par unité de produit que dans les territoires à haute densité animale. Cependant le bilan environnemental des territoires herbagers est meilleur du fait des plus faibles densités animales (au total 34\% du cheptel européen sur $31 \%$ de la SAU, Hercule et al 2017), de la séquestration du carbone par les prairies, et d'impacts locaux (eutrophisation 
Figure 3. Bouquet de services correspondant aux territoires herbagers (légende en figure 2).

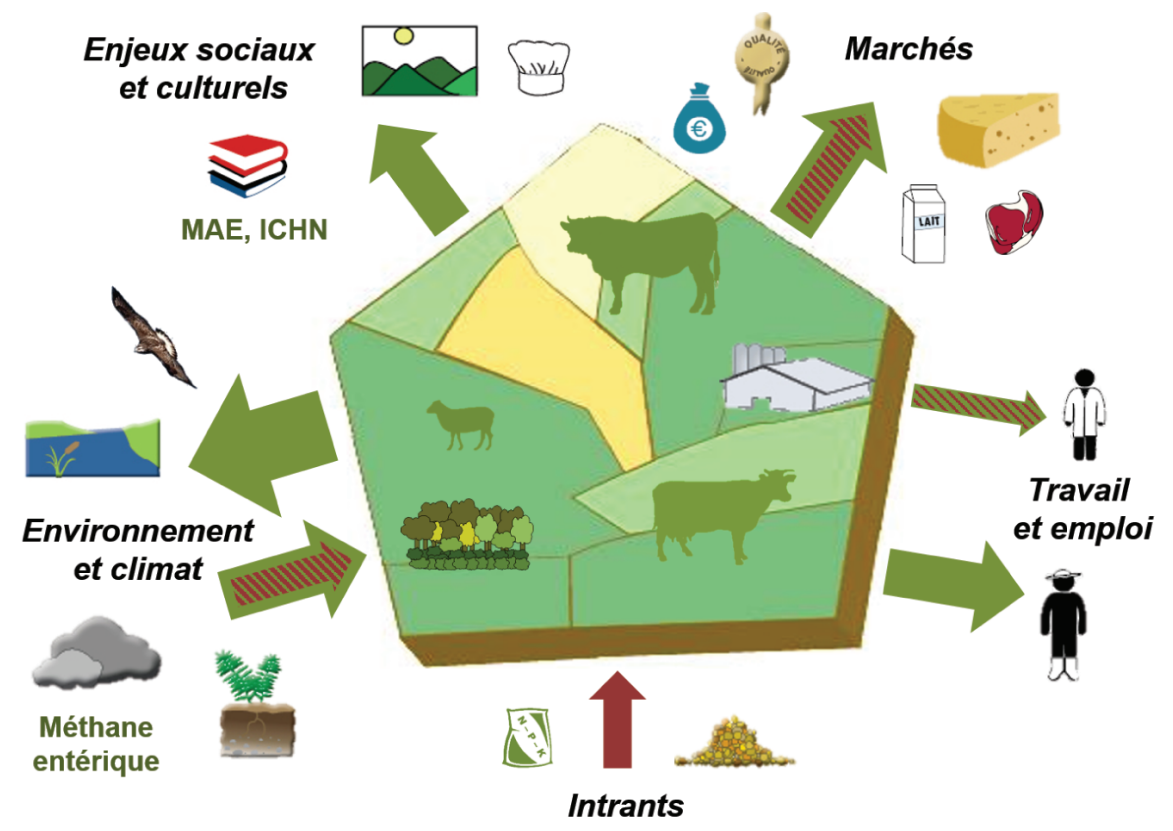

Tableau 2. Atouts et impacts associés aux territoires herbagers.

\begin{tabular}{|c|c|c|}
\hline Interfaces étudiées & Effets positifs & Effets négatifs \\
\hline Intrants & - Niveau d'autonomie fourragère élevée & $\begin{array}{l}\text { - Effets délocalisés de la production } \\
\text { d'aliments concentrés } \\
\text { - Fertilisation minérale des prairies }\end{array}$ \\
\hline Travail et emploi & $\begin{array}{l}\text { - Emplois directs en élevage assez nombreux } \\
\text { (petites structures) } \\
\text { - Maintien du tissu rural } \\
\text { - Emplois indirects (tourisme, filières } \\
\text { de transformation...) }\end{array}$ & - Organisation variable des filières \\
\hline Marchés & $\begin{array}{l}\text { - Produits avec un potentiel de valeur ajoutée } \\
\text { (signe officiel d'identification de la qualité et } \\
\text { de l'origine, circuits courts) }\end{array}$ & $\begin{array}{l}\text { - Saisonnalité de la production à l'herbe } \\
\text { - Finition parfois limitée dans le territoire } \\
\text { (broutards) }\end{array}$ \\
\hline $\begin{array}{l}\text { Enjeux sociaux } \\
\text { et culturels }\end{array}$ & $\begin{array}{l}\text { - Bonne image des systèmes } \\
\text { et des produits } \\
\text { - Qualité nutritionnelle des produits } \\
\text { à l'herbe } \\
\text { - Consentement à payer du consommateur } \\
\text { - Esthétique des paysages } \\
\text { - Patrimoine gastronomique et culturel }\end{array}$ & \\
\hline $\begin{array}{l}\text { Environnement } \\
\text { et climat }\end{array}$ & $\begin{array}{l}\text { - Biodiversité (prairies permanentes, } \\
\text { infrastructures paysagères) } \\
\text { - Séquestration du carbone par prairies } \\
\text { - Préservation de la qualité des eaux }\end{array}$ & $\begin{array}{l}\text { - Émissions élevées de } \mathrm{CH}_{4} \text { par kg } \\
\text { de produit } \\
\text { - Sensibilité aux aléas climatiques } \\
\text { - Poids des « dys-services » (prédation, rats } \\
\text { taupiers) }\end{array}$ \\
\hline
\end{tabular}

des milieux aquatiques) en général limités. Les systèmes qui ont une autonomie fourragère élevée limitent leurs charges opérationnelles (ce qui accroît le revenu) et montrent de très gros atouts dans le cadre de la prise en compte des externalités positives. Leur conception repose sur un paradigme qui se fonde sur la diversification des productions et la valorisation des services écosystémiques, qui sont au cœur de la transition agroécologique. Les possibilités de bien valoriser les produits tout en conservant un haut niveau d'exigence environnementale sont accrues lorsque les modes de commercialisation tirent parti des dimensions non productives de l'élevage ; c'est le cas pour l'agriculture biologique (Benoit et Méda 2017) et pour les AOP qui lient contractuellement les produits à des dimensions 
socio-culturelles et environnementales. L'organisation et la gouvernance des filières prennent alors une importance majeure, et ceci indépendamment des volumes produits comme l'illustre l'exemple des AOP Comté et Laguiole (Barjolle et al 2007, Vollet et al 2017), et favorisent ou non l'emploi local.

Là où les densités animales sont les plus faibles, l'élevage entretient des écosystèmes à haute valeur naturelle comme les zones humides, les pelouses sèches (Lemauviel-Lavenant et Sabatier 2017) ou les alpages (Vollet et al 2017), et les paysages patrimoniaux qui leur sont associés. Les rendements fourragers, et par voie de conséquence la productivité des systèmes, sont en général plus faibles ce qui explique leur dépendance aux aides publiques. À l'autre extrémité du gradient de densité animale, le modèle agro-exportateur irlandais, qui tire parti de conditions très favorables à la pousse de l'herbe, a des performances technico-économiques élevées (autonomie alimentaire, coût de production le plus bas d'Europe) et attire de jeunes éleveurs bien formés vers le secteur laitier (Delaby et al 2017). L'élevage valorise des prairies qui stockent du carbone et contribuent au paysage culturel irlandais, mais l'accroissement de la productivité à l'hectare accroît les émissions brutes de GES, et fait courir un risque accru de lixiviation des nitrates et d'érosion de la biodiversité.

À l'échelle parcellaire, la conduite des prairies constitue un levier majeur pour concilier performances productive et environnementale. Soussana et Lemaire (2014) ont montré que les variables productives et environnementales n'étaient pas maximisées au même niveau d'intensification d'une prairie pâturée, ce qui conduit à rechercher des compromis. La séquestration du carbone et le bilan des émissions de GES par unité de surface atteignent leur optimum à des niveaux d'intensification assez bas, les GES émis par les animaux étant alors compensés par l'augmentation de la teneur en matière organique du sol. La poursuite du processus d'intensification réduit la teneur en matière organique du sol alors que les émissions de GES, et à un niveau moindre le lessivage des nitrates, augmentent. Au-delà d'un certain seuil d'intensification, on assiste à un déstockage massif du carbone. Les quantités de viande ou de lait produites par hectare continuent d'augmenter, mais au détriment de l'ensemble des fonctions écologiques des prairies. Leur restauration passe par une diminution au moins temporaire du chargement, et un meilleur couplage des cycles du carbone et de l'azote. Celui-ci bénéficie de la fixation symbiotique des légumineuses et d'une augmentation de la diversité fonctionnelle (profondeurs de racines...) des prairies permanentes (Frankow-Lindberg et al 2009). Par ailleurs, la gestion des périodes de pâturage, avec par exemple un premier pâturage tardif, augmente l'intensité de floraison, la valeur esthétique des prairies, et la diversité des insectes qu'elles abritent (Milberg et al 2016), sans toutefois réduire le chargement, ni affecter les performances animales (Ravetto Enri et al 2017).

L'échelle du paysage offre d'autres leviers pour concilier les performances productive et environnementale des territoires en jouant sur l'agencement spatial des différents types d'usage agricole (Sabatier et al 2014). Les haies contribuent à l'esthétique des paysages (et éventuellement à la production d'énergie), mais l'entretien d'un réseau de haies représente un coût (et du temps de travail) pour les éleveurs et les collectivités territoriales. Un schéma d'aménagement peu coûteux consiste à connecter les haies existantes par de nouvelles haies orthogonales aux premières afin d'améliorer à la fois leur fonction de corridor écologique, la perception visuelle du bocage et sa valeur culturelle (Groot et al 2010).

\section{3 / D'autres voies de recherche des compromis dans les territoires de polyculture-élevage}

Les territoires de polyculture-élevage peuvent fournir de nombreux services, dès lors qu'une réelle intégration entre cultures, élevages et prairies permet de boucler les cycles biogéochimiques, et en particulier de re-coupler les cycles du carbone et de l'azote (Soussana et Lemaire 2014). Ces territoires concernent $25 \%$ du cheptel européen et $30 \%$ de la SAU (Hercule et al 2017). Dans l'idéal, le lien au sol est fort : les animaux sont le plus possible nourris à partir de prairies temporaires en rotation avec les cultures, et avec des céréales ou des protéagineux produits localement. Leurs déjections fertilisent les terres cultivées ce qui réduit le recours à la fertilisation minérale. La mosaïque de prairies et de cultures contribue à la fourniture de services intrants aux cultures (qualité des sols, fixation d'azote par les légumineuses...) et à l'esthétique des paysages. Ainsi, les systèmes de polyculture-élevage représentent-ils un modèle agroécologique (Bonaudo et al 2014) et éco-efficient (Wilkins 2008) d'agriculture, qui permet de hauts niveaux de production par unité de surface tout en limitant le recours aux aliments importés et les risques de pollution. Ces systèmes jouent également un rôle important dans l'adaptation de l'élevage au changement climatique (Rojas-Downing et al 2017). Une réelle synergie entre les ateliers d'élevage et de cultures s'avère toutefois nécessaire pour concilier performances économiques et environnementales, tant pour l'agriculture biologique (Liu et al 2016) qu'en agriculture conventionnelle (Ryschawy et al 2012, Moraine et al 2014).

Un bouquet de services moyen est présenté figure 4 (et tableau 3), mais ce potentiel n'est pas toujours atteint du fait de la spécialisation croissante en grandes cultures, jusqu'ici plus rentables économiquement et qui requièrent moins de travail. Des variations autour de ce bouquet de services s'observent donc selon la dynamique que conserve l'élevage dans les territoires (Ryschawy et al 2017). Ainsi, dans le Montmorillonnais, de moins en moins d'agriculteurs maintiennent une activité d'élevage et les

Figure 4. Bouquets de services correspondant aux territoires de polyculture-élevage (légende en figure 2).

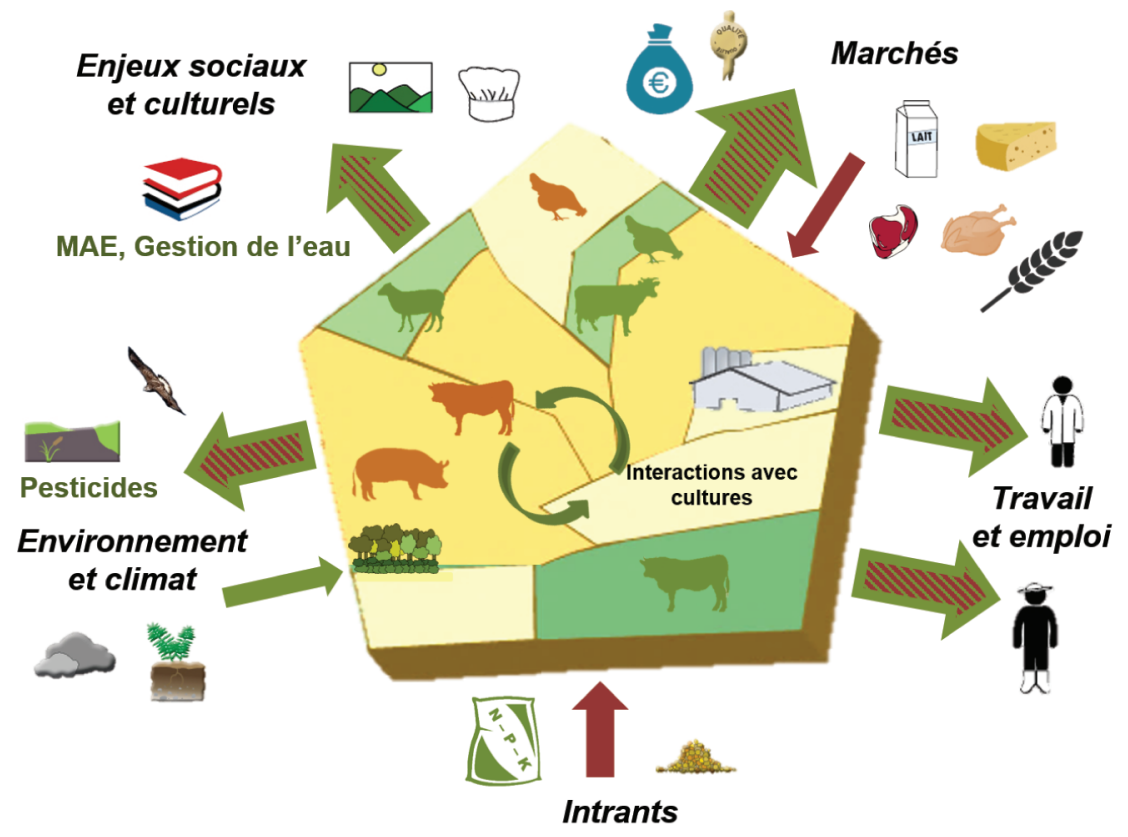


Tableau 3. Atouts et impacts associés aux territoires de polyculture-élevage.

\begin{tabular}{|c|c|c|}
\hline Interfaces étudiées & Effets positifs & Effets négatifs \\
\hline Intrants & $\begin{array}{l}\text { - Production locale des aliments du bétail } \\
\text { lorsqu'il y a une bonne intégration entre } \\
\text { cultures et élevages }\end{array}$ & $\begin{array}{l}\text { - Fertilisation minérale des prairies et } \\
\text { pesticides utilisés sur les cultures } \\
\text { - Effets délocalisés de la production } \\
\text { de concentrés }\end{array}$ \\
\hline Travail et emploi & $\begin{array}{l}\text { - Diversité des filières d'élevage sous signes } \\
\text { de qualité qui dynamisent l'emploi dans la } \\
\text { transformation et la commercialisation }\end{array}$ & $\begin{array}{l}\text { - Charge de travail plus élevée en élevage } \\
\text { par rapport aux cultures } \\
\text { - Pénibilité du travail (abattoirs, gavage) }\end{array}$ \\
\hline Marchés & $\begin{array}{l}\text { - Production de masse peu valorisée, } \\
\text { mais stabilisée par la diversification des } \\
\text { productions } \\
\text { - Produits locaux de qualité (volailles, } \\
\text { fromages...) }\end{array}$ & $\begin{array}{l}\text { - Dépendance vis-à-vis de la conjoncture } \\
\text { internationale }\end{array}$ \\
\hline $\begin{array}{l}\text { Enjeux sociaux et } \\
\text { culturels }\end{array}$ & $\begin{array}{l}\text { - Patrimoine gastronomique } \\
\text { - Esthétique des paysages, tourisme } \\
\text { - Bonne image des produits }\end{array}$ & $\begin{array}{l}\text { - Conflits pour la gestion de l'eau (maïs } \\
\text { ensilage) } \\
\text { - Nuisances olfactives }\end{array}$ \\
\hline $\begin{array}{l}\text { Environnement et } \\
\text { climat }\end{array}$ & $\begin{array}{l}\text { - Biodiversité (mosaïque paysagère) } \\
\text { - Effet favorable de la prairie sur la qualité } \\
\text { des eaux et la séquestration du carbone } \\
\text { - Structure des sols } \\
\text { - Services intrants fournis par les prairies }\end{array}$ & $\begin{array}{l}\text { - Pollution des eaux de surface et des nappes } \\
\text { par azote et pesticides (cultures) }\end{array}$ \\
\hline
\end{tabular}

prairies sont menacées. Leur abandon conduit à un appauvrissement en matière organique des sols et à une simplification des paysages. Au contraire, là où l'élevage conserve une place significative, la diversité des habitats est favorable à la biodiversité et les problèmes environnementaux sont moindres. Dans le bassin Tarn-Aveyron et en Bresse, les acteurs locaux mettent en avant la renommée de leurs filières de qualité associées à des élevages de volailles nourries avec du maïs local (oies et canards gras dans le bassin Tarn-Aveyron, poulets de Bresse). La coexistence de ces systèmes est une richesse en termes d'offre gastronomique et paysagère pour les touristes. Les menaces de nature sociale qui pèsent sur ces territoires résultent d'une charge de travail élevée dans les exploitations de polyculture-élevage (Wilkins 2008, Ryschawy et al 2012), de la complexité des formes d'organisation qui limitent toujours les échanges entre céréaliers et éleveurs, et pour les palmipèdes de risques sanitaires (grippe aviaire) et d'une contestation croissante du gavage dans la population.

Pour favoriser une réelle intégration entre cultures et élevage, différents leviers d'action peuvent être envisagés. L'insertion dans les rotations de légumineuses pures (luzerne : Moraine et al 2016) ou en mélange, ou de cultures de protéagineux fait entrer l'azote atmosphérique dans le système et stabilise le rapport $\mathrm{C} / \mathrm{N}$ de la matière organique du sol. Kragt et Robertson (2014) ont ainsi montré que l'introduction de prairies de luzerne dans la succession culturale augmentait la fertilité des sols et leur capacité drainante. Lorsque la luzerne est présente pendant deux ans dans la rotation, on observe une amélioration conjointe des performances économique et environnementale du système. Au-delà, les bénéfices environnementaux continuent d'augmenter mais le manque à gagner lié au prix élevé des céréales réduit la valeur de ce qui est produit sur l'exploitation, ce qui révèle un compromis.

À l'échelle de petites régions de polyculture-élevage, des cartographies révèlent une nouvelle fois que le service d'approvisionnement est négativement corrélé au service de régulation du climat, avec pour indicateur le stockage du carbone dans le sol (delta du Guadalquivir : Palomo et al 2014, nord de la Belgique : Van der Biest et al 2014). La part et la localisation de l'élevage n'était pas précisée dans ces études. Dans l'étude belge, le service de régulation du climat est très variable là où le service d'approvisionnement est modéré, mais il diminue systématiquement lorsque le service d'approvisionnement augmente, ce qui correspondrait aux zones céréalières intensives d'où les prairies ont disparu (Van der Biest et al 2014). D'autres travaux permettent d'analyser les consé- quences d'une réduction de la fertilisation minérale des prairies temporaires et des cultures, d'une meilleure gestion des déjections animales et d'une restauration des zones humides sur le service de purification de l'eau (bassin AdourGaronne : Maes et al 2012a).

\section{3 / Des scenarios prospectifs mettent en relief les compro- mis entre services aux échelles nationales ou globales}

Plusieurs travaux récents consistent en des scénarios prospectifs qui évaluent les conséquences de différentes options pour nourrir l'humanité tout en cherchant à limiter l'emprise anthropique de l'élevage sur les milieux naturels et ses émissions de GES. Reposant sur des démarches de modélisation globale, ils simulent les effets du changement climatique (Havlik et al 2015, Weindl et al 2015), ou d'un changement des modes de production (Röös et al 2016) et/ou de consommation (Westhoek et al 2014) sur un ensemble de variables de différentes natures: les volumes produits, les modes d'usage des terres, la santé humaine, l'emploi en élevage, etc. Ainsi les sorties de ces modèles révèlent-elles de nouveaux types de bouquets de services. 


\section{1 / Changement climatique et adaptation des systèmes de rumi- nants à l'évolution des rendements fourragers}

L'élevage constitue un secteur clé dans les stratégies de réduction des émissions de GES et d'adaptation au changement climatique (Rojas-Downing et al 2017). Le travail de cartographie des services écosystémiques réalisé par Kirchner et al (2015) en Autriche croise différents scenarios d'évolution de l'agriculture avec quatre scenarios climatiques qui tablent tous sur une augmentation des températures moyennes de $1,5^{\circ} \mathrm{C}$ à $1^{\prime}$ horizon 2040 mais se distinguent par l'intensité et la répartition des précipitations. $\mathrm{La}$ perturbation du régime de précipitations modulerait les niveaux de fourniture des services et conduirait à une intensification des zones les plus productives. La vulnérabilité des systèmes de cultures serait accrue. Une légère augmentation du chargement des prairies d'alpage augmenterait leur productivité, et par voie de conséquence la teneur en matière organique des sols et la contribution des alpages au service de régulation du climat (Smith et al 2005). En revanche, la valeur alimentaire des fourrages baisserait en réponse à des effets directs (e.g. une réduction de la teneur en azote des fourrage lorsque la teneur en $\mathrm{CO}_{2}$ atmosphérique augmente) ou indirects (via des modifications de la structure des communautés prairiales) du changement climatique (Dumont et al 2015).

Ces travaux de modélisation révèlent combien l'évolution des rendements végétaux est stratégique dans les orientations d'élevage en raison de leur poids dans l'alimentation animale. Les rendements en herbe sont estimés de manière très variable selon les modèles de croissance utilisés et les hypothèses d'adaptation des couverts à l'augmentation de la teneur en $\mathrm{CO}_{2}$ atmosphérique. Havlik et al (2015) concluent toutefois qu'à l'échelle mondiale on devrait assister à une augmentation de la part des systèmes herbagers dans l'alimentation des ruminants du fait d'effets du changement climatique en général moins négatifs sur la production d'herbe que sur celle des céréales. Ceux-ci sont toutefois sujets à de fortes variations régionales. À l'inverse de la tendance globale, la productivité du maïs devrait croître en Europe de l'Ouest en réponse à l'augmentation de la teneur en $\mathrm{CO}_{2}$ atmosphérique, alors que la productivité des prairies diminuerait (Weindl et al 2015). Les évolutions de la productivité de l'herbe et du maïs seraient plus parallèles en Europe de l'Est (Weindl et al 2015). L'adaptation au changement climatique par des changements d'usage des terres favoriserait donc plutôt les systèmes de polyculture-élevage en
Figure 5. Évolution de la surface minimale requise pour nourrir une population selon la part des protéines animales dans son régime (adapté de Van Kernebeek et al 2016 et van Zanten et al 2016).

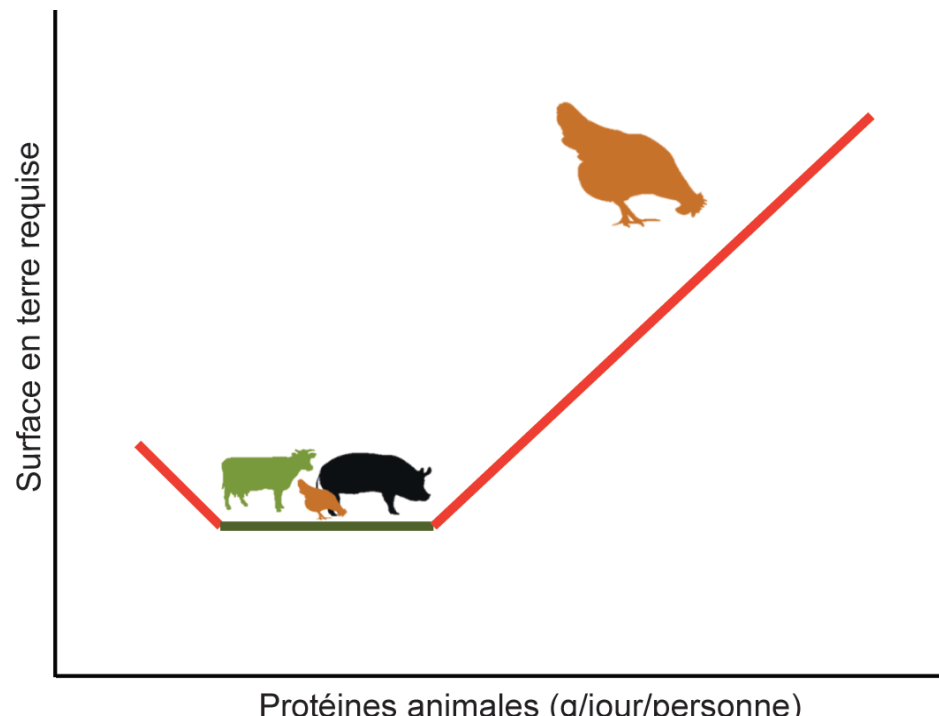

Europe de l'Ouest, mais l'usage des terres resterait stable à l'échelle du continent (Havlik et al 2015).

\section{2 / Réduire la consommation de produits carnés, un levier efficace pour la santé humaine et l'environ- nement}

Plusieurs études récentes simulent les effets d'une forte réduction de la consommation de produits animaux dans l'alimentation (Hedenus et al 2014, Westhoek et al 2014), en invoquant les répercussions négatives d'une consommation excessive de produits carnés sur la santé, et la réticence croissante dans les sociétés occidentales à faire tuer des animaux pour les manger. De tels changements d'habitudes alimentaires réduiraient les émissions de GES de manière sensiblement plus marquée qu'une adaptation des pratiques d'élevage (Popp et al 2010). Ainsi, une réduction de moitié de la consommation de protéines animales par les européens entrainerait une baisse de $40 \%$ des émissions d'azote, et de 25 à $40 \%$ des émissions de GES à l'échelle du continent (Westhoek et al 2014). On assisterait à une réorientation d'une partie des surfaces de production existantes vers l'alimentation humaine (céréales, légumineuses) et les biocarburants. La teneur en acides gras saturés du régime moyen européen serait réduite de $40 \%$, ce qui l'alignerait sur les recommandations de l'OMS.

\section{3 / Les changements de modes de production et de consommation, des leviers pour réduire la compé- tition entre l'alimentation animale et humaine}

Plusieurs scénarios simulent une utilisation de coproduits ou de déchets ali- mentaires pour nourrir les animaux, ce terres entre 1'alimentation animale et l'alimentation humaine (Röös et al 2016, van Zanten et al 2016, zu Ermgassen et al 2016). Tous ces scénarios comportent une hypothèse de réduction de la consommation de produits animaux et une réorientation des productions d'herbivores vers des systèmes plus herbagers, valorisant les terres non utilisables pour des cultures. À l'échelle mondiale, il serait ainsi possible de fournir $21 \mathrm{~g}$ de protéines/j/personne, en accord avec les recommandations de l'OMS (van Zanten et al 2016). En Suède, de tels scénarios réduiraient la production laitière, l'emploi agricole mais également les émissions de GES (Röös et al 2016) ; les besoins en azote et en phosphore, bien que réduits, resteraient toutefois supérieurs aux valeurs recherchées (Rockström et al 2009). Van Kernebeek et al (2016) proposent une courbe « en crosse » qui représente l'emprise de l'agriculture sur les terres selon la quantité de protéines animales dans l'alimentation humaine (figure 5). Il existerait ainsi un intervalle de valeurs de régimes (qui englobe les $21 \mathrm{~g} / \mathrm{j} /$ pers. proposés par van Zanten et al 2016) au sein duquel l'emprise sur les terres serait minimale. En-deçà de cet intervalle, un scénario végétalien ne permet plus de valoriser les coproduits et les prairies non cultivables, et nécessiterait donc plus de terres pour nourrir la population. Dans ces scénarios les monogastriques valorisent les déchets alimentaires et les coproduits des cultures et de l'industrie agro-alimentaire. La production de porc de l'UE réduirait ainsi ses besoins en surface d'un cinquième, sans modifier la vitesse de croissance des animaux, ni la qualité organoleptique de leur viande (zu Ermgassen et al 2016). Une autre option consiste à produire des insectes sur les déchets alimentaires et à qui atténuerait la concurrence pour les 
utiliser ces insectes comme source de protéines pour l'alimentation animale (Stamer 2015). De telles options nécessiteront de mettre en place une réglementation sanitaire stricte pour collecter les déchets, les stocker et les transformer.

En France, les modalités de ce type de transitions sont discutées dans le scénario Afterres2050 (Solagro 2014). Celuici simule les effets d'une réduction de $25 \%$ notre consommation totale en protéines (qui passerait de 90 à $70 \mathrm{~g} / \mathrm{j} /$ personne), et d'une baisse de 40 à $20 \%$ de la part des produits animaux (poissons et crustacés compris) en leur substituant des protéines végétales. Il table aussi sur une réduction de $60 \%$ des gaspillages alimentaires et une augmentation du recyclage. Le recours au système " maïssoja » serait réduit, et la part des systèmes en agriculture biologique, AOP, Label Rouge, et des systèmes herbagers accrue. Sous de telles hypothèses, les productions animales françaises seraient divisées par deux à horizon 2050, avec d'importantes conséquences sur les équilibres territoriaux. Cela aurait en revanche des conséquences bénéfiques pour l'environnement, le climat, et l'emploi, avec 140000 emplois créés en France d'ici 2050 mais de fortes relocalisations.

\section{4 / Intérêt et limites des modé- lisations globales}

Le principal intérêt des démarches prospectives par modélisation réside dans l'effort d'assemblage d'informations de différentes origines qui garantit une cohérence d'ensemble aux projections réalisées. Plus que les autres approches, ces modélisations explorent des scénarios en rupture qui même s'ils apparaissent encore très théoriques, voire hors contexte, permettent de réfléchir à des alternatives très poussées. La transparence de la démarche, avec des hypothèses clairement posées, donne accès à la construction des résultats que l'on peut donc discuter voir remettre en cause (Mahmoud et al 2009). Ces exercices mettent en évidence de nouveaux types de bouquets de services, et des modèles d'optimisation pourraient permettre d'identifier les modes de production fournissant les bouquets les plus équilibrés. La principale limite de ces démarches est qu'elles n'ont jusqu'ici que peu considéré certains risques environnementaux majeurs (e.g. l'augmentation d'usage des pesticides du fait d'une consommation accrue de céréales, de fruits et de légumes), et qu'elles ont occulté les conséquences sociales (e.g. pour l'emploi en élevage et les équilibres territoriaux) induites par ces transitions. Les présupposés sur les transitions alimentaires ne prennent également pas en compte le prix des produits et leur effet sur la demande alimentaire. Enfin, les dimensions sociales ne sont que rarement quantifiées (voir cependant Solagro 2014 et Röös et al 2016), ni les effets de ces transitions sur les services de régulation et les services culturels fournis par l'élevage.

\section{Conclusion}

L'alimentation humaine - au travers de la part des produits animaux - et l'alimentation animale - au travers de son emprise territoriale - apparaissent comme les deux principaux leviers permettant de moduler les impacts environnementaux de l'élevage. Les cartographies de services, tout comme la représentation à l'aide de la grange des bouquets de services rendus par l'élevage dans les territoires, mettent en évidence un antagonisme entre le service d'approvisionnement d'une part, les services culturels et de régulation d'autre part. Il existe toutefois des opportunités pour faire évoluer les systèmes et les territoires vers une meilleure conciliation de leurs performances productive et environnementale. Ces opportunités résultent de la non-linéarité et de la variabilité des relations entre services (Kragt et Robertson 2014, Van der Biest et al 2014), de la prise en compte des temporalités (Ravetto Enri et al 2017), de l'hétérogénéité spatiale (Sabatier et al 2014), et des interactions techniques ou organisationnelles entre les systèmes d'un même territoire (Moraine et al 2014, Ryschawy et al 2017). Ainsi, certains antagonismes qui semblent incontournables à une échelle globale peuvent-ils être solutionnés localement, par le développement de systèmes innovants et la coexistence de différents systèmes.

Dans la littérature, on trouve cependant beaucoup moins de situations « gagnantgagnant » qui concilient performances productive et environnementale que de situations de concurrence. Une métaanalyse récente, qui embrasse une large gamme d'environnements terrestres et littoraux, rapporte trois fois plus de situations de concurrences entre la fourniture de différents services ou entre acteurs, que de situations de synergies (Howe et al 2014). Pour ces auteurs, il est plus aisé d'aboutir à une solution gagnantgagnant lorsqu'on s'affranchit de l'idée que les services d'approvisionnement seraient systématiquement prioritaires par rapport aux autres services écosystémiques. Les solutions gagnant-gagnant sont généralement le résultat d'une négociation collective, plutôt que de situations où primeraient les intérêts individuels et les rapports de force, et ce y compris dans les territoires d'élevage (Groot et al 2010, Lamarque et al 2014, Moraine et al 2016).

Poursuivre l'analyse des bouquets de services fournis par l'élevage devrait permettre de comprendre ce qui est géné- rique ou au contraire propre à certains territoires. Afin de mieux valoriser la diversité des services fournis par l'élevage, trois autres pistes de recherches nécessiteront d'être approfondies : i) la compréhension de l'impact des politiques publiques, ii) la quantification des services et dys-services issus de l'élevage, et iii) l'information apportée au consommateur.

La Politique Agricole Commune intègre de longue date certaines dimensions environnementales et culturelles de l'élevage. Ses effets sont réels dans certains territoires, notamment les zones à handicaps naturels (Cooper et al 2009), mais semblent plus limités ailleurs (Kleijn et al 2006). Une des raisons est probablement le manque de ciblage des critères choisis. Le poids historique du soutien à la production se retrouve dans la répartition des aides directes bien que cellesci sont aujourd'hui découplées de la production. Ainsi, les systèmes intensifs qui utilisent beaucoup d'intrants pour alimenter les animaux et occasionnent le plus de nuisances locales bénéficient-ils indirectement des aides aux grandes cultures, et d'aides à l'investissement et à l'export. Les systèmes ruminants extensifs ne sont quant à eux pas incités à réduire leurs émissions de GES par kilo produit alors que des leviers existent. Plus généralement, la contribution de l'élevage au changement climatique ne donne lieu ni à enregistrement, ni à la fixation de seuils conditionnant l'attribution d'aides ou le paiement de pénalités, comme cela existe par exemple pour la directive Nitrates. Un meilleur ciblage des soutiens publics, en adéquation avec les atouts environnementaux des différents systèmes et la sensibilité des milieux, apparaît donc comme indispensable.

Par ailleurs, la rémunération des externalités positives ou la taxation des externalités négatives de l'élevage reste très lacunaire faute d'information complète accessible aux décideurs publics, de modalités de gouvernance adaptées, et du poids des lobbies. On constate une dissymétrie structurelle des incitations procurées par le marché qui rémunère la production et les politiques publiques qui visent à rémunérer les externalités. Des réflexions sont toutefois engagées pour faire reconnaître et rémunérer les services rendus par l'activité agricole au travers de paiements pour services environnementaux (voir la revue de Duval et al 2016), par exemple dans les filières en agriculture biologique et la filière ovins viande. En parallèle, des travaux ont été initiés pour quantifier les externalités négatives liées à l'usage des pesticides (Bourguet et Guillemaud 2016), mais ceux-ci restent préliminaires et ne concernent qu'assez peu l'élevage (maïs fourrage...). Une meilleure évaluation des dif- 
férents coûts environnementaux de l'élevage (e.g. Chaudhary et Kastner 2016, Hammer et al 2016) et de ses coûts politiques et sociaux (Marshall et Levy 2011) permettrait de mieux cibler les politiques publiques.

Enfin, la diversité des services rendus par les différentes formes d'élevage n'est encore que peu visible du consommateur. Dans un contexte de défiance grandissante vis à vis de l'élevage dit « industriel » (crises sanitaires, remise en cause des conditions de vie des animaux...), une part croissante des consommateurs se tourne vers des produits sous signes de qualité (Benoit et Méda 2017 ; Vollet et al 2017) ou/et en vente directe (Delfosse et al 2017). Ils recherchent alors une relation de confiance avec l'éleveur qui se fonde sur une information détaillée sur ses pratiques d'élevage (alimentation, abattage...). Les attributs des produits animaux qui renforcent le consentement à payer des consommateurs restent à préciser (Dumont et al 2014), mais cette dynamique montre le potentiel de valorisation par le marché des dimensions non productives de l'élevage. La question de l'étiquetage des produits alimentaires fait l'objet d'intenses débats entre associations de consommateurs, industriels, distributeurs et la puissance publique. On assiste actuellement à une explosion de normes, de labels et d'indications publiques ou privées qui risquent de saturer les capacités de discernenement des consommateurs. La récente règlementa- tion européenne INCO vise à standardiser ces démarches et propose des avancées quant à l'indication d'origine et au bien-être animal, y compris pour les produits transformés. Mieux informer le consommateur lui permettra de faire des choix afin de consommer (ou non) des produits animaux en adéquation avec ses valeurs.

\section{Remerciements}

Les auteurs remercient Catherine Donnars, Pierre Dupraz, Françoise Lescourret, Sylvain Pellerin, Guy Richard et deux lecteurs anonymes pour leurs remarques pertinentes sur notre manuscrit.

\section{Références}

Alkemade R., van Oorschot M., Miles L., Nellemann C., Bakkenes M., ten Brink B., 2009. GLOBIO3: A framework to investigate options for reducing global terrestrial biodiversity loss. Ecosystems, 12, 374-390.

Anderies J.M, Janssen M., Ostrom E., 2004. A Framework to analyse the robustness of socioecological systems from an institutional perspective. Ecol. Soc., 9, 1-18

Barjolle D., Reviron S., Sylvander B., 2007. Création et distribution de valeur économique dans les filières de fromages AOP. Écon. Soc., 41, 1507-1524.

Bennett E.M., Peterson G.D., Gordon L.J., 2009. Understanding relationships among multiple ecosystem services. Écol. Lett., 12, 13941404.

Benoit M., Méda B., 2017. Enjeux et atouts des productions animales sous signe officiel de qualité pour répondre aux attentes sociétales. In Numéro spécial, L'élevage en Europe : une diversité de services et d'impacts. Dumont B. (Éd). INRA Prod. Anim. 30, 381-394.

Berkes F., Colding J., Folke C., 2003. Navigating Social-Ecological Systems-Building Resilience for Complexity and Change. Cambridge University Press, 393p.

Bertoni G., Trevisi E., Houdijk J., Calamari L., Athanasiadou S., 2016. Welfare is affected by nutrition through health, especially immune function and inflammation. In: Nutrition and the Welfare of Farm Animals. Phillips C.J.C. (Ed). Springer International Publishing, AG, Switzerland, 85-113.

Bonaudo T., Burlamaqui Bendahan A., Sabatier R., Ryschawy J., Bellon S., Leger F., Magda D., Tichit M., 2014. Agroecological principles for the redesign of integrated croplivestock systems. Europ. J. Agron., 57, 43-51.

Bourguet D., Guillemaud T., 2016. The hidden and external costs of pesticide use. In: Sustainable Agriculture Reviews. Lichtfouse E. (Ed). Springer International Publishing, Switzerland, 35-120.

Brummel R., Nelson K., 2014. Does multifunctionality matter to US farmers? Farmers motiva- tions and conceptions of multifunctionality in dairy systems, J. Environ. Manage., 146, 451-462.

Chaudhary A., Kastner T., 2016. Land use biodiversity impacts embodied in international food trade. Global Environ. Change, 38, 195-204.

Coignard M., Guatteo R., Veissier I., de Boyer des Roches A., Mounier L., Lehébel A., Bareille N., 2013. Description and factors of variation of the overall health score in French dairy cattle herds using the Welfare Quality $\AA$ assessment protocol. Prev. Vet. Med., 112, 296308 .

Cooper T., Hart K., Baldock D., 2009. The provision of public goods through agriculture in the European Union. Report for DG Agriculture and Rural Development, Contract No 30-CE0233091/00-28, Institute for European Policy, London, 396p.

Delaby L., Fiorelli J.L., 2014. Élevages laitiers à bas intrants : entre traditions et innovations. In : Numéro spécial, quelles innovations pour quels systèmes d'élevage? Ingrand S., Baumont R. (Éd). INRA Prod. Anim., 27, 123-133.

Delaby L., Chatellier V., Horan B., Dumont B. 2017. L'Irlande, un territoire porté par l'élevage laitier dans des conditions de milieu favorable et de marchés incertains. In : Numéro spécial, L'élevage en Europe : une diversité de services et d'impacts. Dumont B. (Éd). INRA Prod. Anim. 30, 321-332.

Delfosse C., Dumont B., Hostiou N., 2017. Des services contrastés rendus par l'élevage dans les espaces urbains et périurbains européens. In : Numéro spécial, L'élevage en Europe : une diversité de services et d'impacts. Dumont B. (Éd). INRA Prod. Anim. 30, 395-406.

Dick J., Andrews C., Beaumont D.A., Benham S., Brooks D.R., Corbett S., Lloyd D., McMillan S., Monteith D.T., Pilgrim E.S., Rose R., Scott A., Scott T., Smith R.I., Taylor C., Taylor M. Turner A., Watson H., 2011. A comparison of ecosystem services delivered by 11 long-term monitoring sites in the UK environmental change network. Environmetrics, 22, 639-648.

Dourmad J.Y., Ryschawy J., Trousson T., Bonneau M., Gonzalez J., Houwers H.W.J., Hviid M., Zimmer C., Nguyen T.L.T., Morgensen
L., 2014. Evaluating environmental impacts of contrasting pig farming systems with life cycle assessment. Animal, 8, 2027-2037.

Dourmad J.Y., Delaby L., Boixadera J., Ortis C., Méda M., Gaigné C., Dumont B., 2017. Diversité des services rendus par les territoires à forte densité d'élevage, trois cas d'étude en Europe. In : Numéro spécial, L'élevage en Europe : une diversité de services et d'impacts. Dumont B. (Éd). INRA Prod. Anim. 30, 303320 .

Dumont B., González-García E., Thomas M., Fortun-Lamothe L., Ducrot C., Dourmad J.Y., Tichit M., 2014. Forty research issues for the redesign of animal production systems in the $21^{\text {st }}$ century. Animal, 8, 1382-1393.

Dumont B., Andueza D., Niderkorn V., Lüscher A., Porqueddu C., Picon-Cochard C., 2015. A meta-analysis of climate change effects on forage quality in grasslands: specificities of mountain and Mediterranean areas. Grass For. Sci., 70, 239-254.

Dumont B., Dupraz P., Sabatier R., Hercule J., Donnars C., 2017. Une expertise scientifique collective analyse les rôles, impacts et services issus des élevages en Europe. Fourrages, 229, 63-76.

Duru M., Therond O., 2015. Livestock system sustainability and resilience in intensive production zones: which form of ecological modernization? Reg. Environ. Change, 15, 1651-1665.

Duru M., Donnars C., Ryschawy J., Therond O., Dumont B., 2017. La « grange » : un cadre conceptuel pour appréhender les bouquets de services rendus par l'élevage dans les territoires. In : Numéro spécial, L'élevage en Europe : une diversité de services et d'impacts. Dumont B. (Éd). INRA Prod. Anim. 30, 273-284.

Duval L., Binet T., Dupraz P., Lepaly S., Etrillard C, Pech M., Deniel E., Laustriat M., 2016. Paiements pour services environnementaux et méthodes d'évaluation économique. Enseignements pour les mesures agro-environnementales de la politique agricole commune. Étude ${ }^{\circ}$ SSP-2014-017. 21p.

FAO, 2006. Livestock's Long Shadow-Environmental Issues and Options. FAO, Rome, 390p. 
Frankow-Lindberg B.E., Brophy C., Collins R.P., Connolly J., 2009. Biodiversity effects on yield and unsown-species invasion in a temperate forage ecosystem. Ann. Bot.-London, 103, 913-921.

Gerber P.J., Vellinga T., Opio C., Steinfeld H., 2011. Productivity gains and greenhouse gas emissions intensity in dairy systems. Livest. Sci., 139, 100-108.

Groot J.C.J., Jellema A., Rossing W.A.H., 2010. Designing a hedgerow network in a multifunctional agricultural landscape: Balancing trade-offs among ecological quality, landscape character and implementation costs. Europ. J. Agron., 32, 112-119.

Hamann M., Biggs R., Reyers B., 2015. Mapping social-ecological systems: Identifying 'green-loop' and 'red-loop' dynamics based on characteristic bundles of ecosystem service use. Global Environ. Change, 34, 218-226.

Hammer T.J., Fierer N., Hardwick B., Simojoki A., Slade E., Taponen J., Viljanen H., Roslin T., 2016. Treating cattle with antibiotics affects greenhouse gas emissions, and microbiota in dung and dung beetles. Proc. R. Soc. B, 283, 20160150 .

Havlik P.D., Leclère D., Valin H., Herrero M., Schmid E., Soussana J.F., Müller C., Obersteiner M., 2015. Global climate change, food supply and livestock production systems: A bioeconomic analysis. In: Climate change and food systems: global assessments and implications for food security and trade. Elbehri A. (Ed). FAO, Rome, 176-208.

Hedenus F., Wirsenius S., Johansson D., 2014. The importance of reduced meat and dairy consumption for meeting stringent climate change targets. Clim. Change, 124, 79-91.

Hercule J., Chatellier V., Piet L., Dumont B., Benoit M., Delaby L., Donnars C., Savini I., Dupraz P., 2017. Une typologie pour représenter la diversité des territoires d'élevage en Europe. In : Numéro spécial, L'élevage en Europe : une diversité de services et d'impacts. Dumont B. (Éd). INRA Prod. Anim., 30, 285-302.

Howe C., Suich H., Vira B., Mace G.M., 2014. Creating win-wins from trade-offs? Ecosystem services for human well-being: A meta-analysis of ecosystem service trade-offs and synergies in the real world. Global Environ. Change, 28, 263-275.

Jopke C., Kreyling J., Maes J., Koellner T., 2015. Interactions among ecosystem services across Europe: Bagplots and cumulative correlation coefficients reveal synergies, trade-offs, and regional patterns. Ecol. Indic., 49, 46-52.

Kirchner M., Schmidt J., Kindermann G., Kulmer V., Mitter H., Prettenthaler F., Rüdisser J., Schauppenlehner T., Schönhart M., Strauss F., Tappeiner U., Tasser E., Schmid E., 2015. Ecosystem services and economic development in Austrian agricultural landscapes - The impact of policy and climate change scenarios on trade-offs and synergies. Ecol. Econ., 109, 161-174.

Kleijn D., Baquero R.A., Clough Y., Díaz M., De Esteban J., Fernández F., Gabriel D., Herzog F., Holzschuh A., Jöhl R., Knop E., Kruess A., Marshall E.J.P., Steffan-Dewenter I., Tscharntke T., Verhulst J., West T.M., Yela J.L., 2006. Mixed biodiversity benefits of agrienvironment schemes in five European countries. Ecol. Lett., 9, 243-254.
Kragt M.E., Robertson M.J., 2014. Quantifying ecosystem services trade-offs from agricultural practices. Ecol. Econ., 102, 147-157.

Kroll F., Muller F., Haase D., Fohrer N., 2012. Rural-urban gradient analysis of ecosystem services supply and demand dynamics. Land Use Policy, 29, 521-535.

Lamarque P., Meyfroidt P., Nettier B., Lavorel S., 2014. How ecosystem services knowledge and values influence farmers' decision making. PLoS ONE, 9, e107572

Larue R., 2015. Le végétarisme et ses ennemis vingt-cinq siècles de débats. Presses Universitaires de France, Paris, France, 306p.

Lele S., Springate-Baginski O., Lakerveld R., Deb D., Dash P., 2013. Ecosystem services: Origins, Contributions, Pitfalls, and Alternatives. Conserv. Soc., 11, 343-358.

Lemauviel-Lavenant S., Sabatier R., 2017. Quand l'élevage est garant de la conservation de milieux patrimoniaux. In : Numéro spécial, L'élevage en Europe : une diversité de services et d'impacts. Dumont B. (Éd). INRA Prod. Anim. 30, 351-362.

Lescourret F., Magda D., Richard G., AdamBlondon A.F., Bardy M., Baudry J., Doussan I., Dumont B., Lefevre F., Litrico I., MartinClouaire R., Montuelle B., Pellerin S., Plantegenest M., Tancoigne E., Thomas A. Guyomard H., Soussana J.F., 2015. A socialecological approach to managing multiple agroecosystem services. Curr. Opin. Environ. Sustain. $14,68-75$.

Liu H., Meng J., Bo W., Cheng D., Li Y., Guo L., Li C., Zheng Y., Liu M., Ning T., Wu G., Yu X., Feng S., Wuyun T., Li J., Li L., Zeng Y., Liu S.V., Jiang G., 2016. Biodiversity management of organic farming enhances agricultural sustainability. Sci. Rep., 6, 23816.

Loyon L., Burton C.H., Misselbrook T., Webb J., Philippe F.X., Aguilar M., Doreau M., Hassouna M., Veldkamp T., Dourmad J.Y., Bonmati A., Grimm E., Sommer S.G., 2016. Best available technology for European livestock farms: Availability, effectiveness and uptake. J. Environ. Manage., 166, 1-11.

Maes J., Egoh B., Willemen L., Liquete C., Vihervaara P., Schägner J.P., Grizzetti B. Drakou E.G., La Notte A., Zulian G., Bouraoui F., Luisa Paracchini M., Braat L., Bidoglio G., 2012a. Mapping ecosystem services for policy support and decision making in the European Union. Ecosyst. Serv., 1, 31-39.

Maes J., Paracchini M.L., Zulian G., Dunbar M.B., Alkemade R., 2012b. Synergies and trade-offs between ecosystem service supply, biodiversity, and habitat conservation status in Europe. Biol. Conserv., 155, 1-12.

Mahmoud M., Liu Y., Hartmann H., Stewart S. Wagener T., Semmens D., Stewart R., Gupta H., Dominguez D., Dominguez F., Hulse D., Letcher R., Rashleigh B., Smith C., Street R., Ticehurst J., Twery M., van Delden H., Waldick R., White D., Winter L., 2009. A formal framework for scenario development in support of environmental decision-making. Environ. Modell. Softw., 24, 798-808.

Marshall B.M., Levy S.B., 2011. Food animals and antimicrobials: Impacts on human health. Clin. Microbiol. Rev., 24, 718-733.
MEA - Millennium Ecosystem Assessment, 2005. Rapport de synthèse de l'Évaluation des Écosystèmes pour le Millénaire 59p.

Milberg P., Bergman K.O., Cronvall E., Eriksson Å.I, Glimskär A., Islamovic A., Jonason D., Löfqvist Z., Westerberg L., 2016. Flower abundance and vegetation height as predictors for nectar-feeding insect occurrence in Swedish semi-natural grasslands. Agricult. Ecosys. Environ., 230, 47-54.

Mollard A., Rambonilaza M., Vollet D., 2006. Aménités environnementales et rente territoriale sur un marché de services différenciés : le cas du marché des gîtes ruraux labellisés en France, Rev. Éco. Pol., 116, 251-276.

Moraine M., Duru M., Nicholas P., Leterme P., Therond O., 2014. Farming system design for innovative crop-livestock integration in Europe. Animal, 8, 1204-1217.

Moraine M., Grimaldi J., Murgue C., Duru M., Therond O., 2016. Co-design and assessment of cropping systems for developing crop-livestock integration at the territory level. Agricult. Sys., 147, 87-97.

Palomo I., Martín-López B., Alcorlo P., Montes C., 2014. Limitations of protected areas zoning in Mediterranean cultural landscapes under the ecosystem services approach. Ecosystems, 17 1202-1215.

Patience J.F., Rossoni-Serão M.C, Gutiérrez N.A., 2015. A review of feed efficiency in swine: biology and application. J. Anim. Sci. Biotechnol., 6, 33 .

Picard P., 1994. Éléments de microéconomie : théorie et applications. Montchrestien, Paris, $587 \mathrm{p}$.

Popp A., Lotze-Campen H., Bodirsky B., 2010. Food consumption, diet shifts and associated non- $\mathrm{CO}_{2}$ greenhouse gases from agricultural production. Global Environ. Change, 20, 451462.

Raudsepp-Hearne C., Peterson G.D., Bennett E.M., 2010. Ecosystem service bundles for analyzing tradeoffs in diverse landscapes. Proc. Natl. Acad. Sci., 107, 5242-5247.

Ravetto Enri S., Probo M., Farruggia A., Lanore L., Blanchetete A., Dumont B., 2017. A biodiversity-friendly rotational grazing system enhancing flowering-visiting insect assemblages while maintaining animal and grassland productivity. Agricult. Ecosys. Environ., 241, $1-10$.

Rockström J., Steffen W., Noone K., Persson $\AA$, Stuart Chapin F., Lambin E.F., Lenton T.M., Scheffer M., Folke C., Schellnhuber H.J., Nykvist B., de Wit C.A., Hughes T., van der Leeuw S., Rodhe H., Sörlin S., Snyder P.K., Costanza R., Svedin U., Falkenmark M., Karlberg L., Corell R.W., Fabry V.J., Hansen J., Walker B., Liverman D., Richardson K., Crutzen P., Foley J.A., 2009. A safe operating space for humanity. Nature, 461, 472-475.

Rodriguez J.P., Beard Jr. T.D., Bennett E.M., Cumming G.S., Cork S.J., Agard J., Dobson A.P., Peterson G.D., 2006. Trade-offs across space, time, and ecosystem services. Ecol. Soc., 11,28 .

Rodríguez-Ortega T., Oteros-Rozas E., RipollBosch R., Tichit M., Martín-López B., Bernués A., 2014. Applying the ecosystem services fra- 
mework to pasture-based livestock farming systems in Europe. Animal, 8, 1361-1372.

Roguet C., Gaigné C., Chatellier V., Cariou S., Carlier M., Chenu R., Daniel K., Perrot C., 2015. Spécialisation territoriale et concentration des productions animales européennes : état des connaissances et facteurs explicatifs. INRA Prod. Anim., 28, 5-22.

Rojas-Downing M.M., Nejadhashemi A.P. Harrigan T., Woznicki S.A., 2017. Climate change and livestock: Impacts, adaptation, and mitigation. Climate Risk Manage., 16, 145-163.

Röös E., Patel M., Spångberg J., Carlsson G., Rydhmer L., 2016. Limiting livestock production to pasture and by-products in a search for sustainable diets. Food Policy, 58, 1-13.

Ryschawy J., Choisis N., Choisis J.P., Joannon A., Gibon A., 2012. Mixed crop-livestock systems: an economic and environmental-friendly way of farming? Animal, 6, 1722-1730.

Ryschawy J., Tichit M., Bertrand S., Allaire G., Plantureux S., Aznar O., Perrot C., Guinot C., Josien E., Lasseur J., Aubert C., Tchakerian E., Disenhaus C., 2015. Comment évaluer les services rendus par l'élevage? Une approche méthodologique sur le cas de la France. INRA Prod. Anim., 28, 23-38.

Ryschawy J., Benoit M., Hostiou N, Delfosse C. 2017. Quelles concurrences et synergies entre cultures et élevage dans les territoires de polyculture-élevage ? In : Numéro spécial, L'élevage en Europe : une diversité de services et d'impacts. Dumont B. (Éd). INRA Prod. Anim. 30, 363-380.

Sabatier R., Doyen L., Tichit M., 2014. Heterogeneity and the trade-off between ecological and productive functions of agro-landscapes: A model of cattle-bird interactions in a grassland agroecosystem. Agricult. Sys., 126, 38-49.
Salou T., Le Mouel C., van der Werf H.M.G., 2017. Environmental impacts of dairy system intensification: the functional unit matters J. Cleaner Prod., 140, 445-454.

Smith J., Smith P., Wattenbach M., Zaehle S. Hiederer R., Jones R.J.A., Montanarella L. Rounsevell M.D.A., Reginster I., Ewert F., 2005. Projected changes in mineral soil carbon of European croplands and grasslands, 19902080. Global Change Biol., 11, 2141-2152.

Solagro 2014. Afterres 2050. Un scénario soutenable pour l'agriculture et l'utilisation des terres en France à l'horizon 2050, Solagro Éd., Toulouse, France, 63p.

Soussana J.F., Lemaire G., 2014. Coupling carbon and nitrogen cycles for environmentally sustainable intensification of grasslands and crop-livestock systems. Agricult. Ecosys. Environ., 190, 9-17.

Stamer A., 2015. Insect proteins-a new source for animal feed. EMBO reports, 16, 676-680.

Tancoigne E., Barbier M., Cointet J.P., Richard G., 2014. The place of agricultural sciences in the literature on ecosystem services. Ecosys Serv., 10, 35-48.

Turner K.G., Odgaard M.V., Bocher P.K. Dalgaard T., Svenning J.C., 2014. Bundling ecosystem services in Denmark: Trade-offs and synergies in a cultural landscape. Landscape Urban Plan., 125, 89-104.

Van der Biest K., D’Hondt R., Jacobs S., Landuyt D., Staes J., Goethals P., Meire P., 2014. EBI An index for delivery of ecosystem service bundles. Ecol. Indic., 37, 252-265.

Van Kernebeek H.R.J., Oosting S.J., Van Ittersum M.K., Bikker P., De Boer I.J.M., 2016. Saving land to feed a growing population consequences for consumption of crop and livestock products. Int. J. Life Cycle Assess., 21, 677-687.

van Oudenhoven A., Petz K., Alkemade R. Hein L., de Groot R, 2012. Framework for systematic indicator selection to assess effects of land management on ecosystem services. Ecol. Indic., 21, 110-122.

van Zanten H.H.E., Meerburg B.G., Bikker P., Herrero M., de Boer I.J.M., 2016. Opinion paper: The role of livestock in a sustainable diet: a land-use perspective. Animal, 10, 547 549 .

Vollet D., Huguenin-Elie O., Martin B., Dumont B., 2017. La diversité des services rendus par les territoires d'élevage herbagers fournissant des produits de qualité dans des environnements préservés. In : Numéro spécial, L'élevage en Europe : une diversité de services et d'impacts. Dumont B. (Éd). INRA Prod. Anim. 30, 333-350.

Weindl I., Lotze-Campen H., Popp A., Müller C., Havlik P., Herrero M., Schmitz C., Rolinski S., 2015. Livestock in a changing climate: production system transitions as an adaptation strategy for agriculture. Environ. Res. Lett., 10, 094021 .

Westhoek H., Lesschen J.P., Rood T., Wagner S., De Marco A., Murphy-Bokern D., Leip A., van Grinsven H., Sutton M.A., Oenema O., 2014. Food choices, health and environment: Effects of cutting Europe's meat and dairy intake. Global Environ. Change, 26, 196-205.

Wilkins R.J., 2008. Eco-efficient approaches to land management: a case for increased integration of crop and animal production systems. Philos. T.R. Soc. B, 363, 517-525.

zu Ermgassen E.K.H.J., Phalan B., Green R.E., Balmford A., 2016. Reducing the land use of EU pork production: where there's swill, there's a way. Food Policy, 58, 35-48.

\section{Résumé}

L'élevage représente une activité majeure dans nombre de territoires ruraux européens. Au-delà des controverses sur les conséquences environnementales de l'élevage, et sur les conditions de vie et d'abattage des animaux, il rend différents services à la société qu'il convient de révéler, et si possible de quantifier. Le cadre conceptuel des bouquets de services permet de considérer la coexistence d'effets positifs et négatifs de l'élevage à un instant donné sur un territoire. L'augmentation d'un service impliquant en général la diminution d'autres, ce cadre permet d'identifier les compromis à considérer au sein des territoires. Aux échelles larges (de la petite région agricole à l'Europe), on constate souvent un antagonisme entre production et emploi d'une part, services environnementaux et culturels d'autre part. Les cartographies de services révèlent l'effet des systèmes dominants dans les territoires, mais ne prennent pas en compte la coexistence avec des systèmes de niche, dont les contributions positives sont largement masquées. Une analyse à des échelles plus fines (bassin versant, canton, exploitation) montre qu'il est possible de dépasser ces antagonismes en prenant en compte les temporalités, l'hétérogénéité spatiale, et les interactions techniques ou organisationnelles entre différents types de systèmes d'élevage (et de cultures) d'un même territoire. Des modélisations aux échelles larges permettent de simuler de manière prospective les conséquences du changement climatique et d'évolutions plus tranchées des modes de production et de consommation. L'alimentation humaine - au travers de la part des protéines d'origine animale - et l'alimentation animale - du fait de son impact sur l'usage des terres - ont un poids décisif dans ces scénarios. Nous concluons en proposant des pistes de recherches à approfondir afin de mieux valoriser la diversité des services fournis par l'élevage.

\section{Abstract}

\section{Bundles of services make it possible to identify trade-offs and options for livestock production areas}

Livestock is a major actor of rural landscape and economics across Europe. It has however focused various controversies as regards to its environmental impacts, and the consequences of industrial production systems on animal welfare. Bundles of services account for both livestock positive and negative effects that simultaneously occur within territories. Mapping ecosystem services at a large scale (from landscape to the European Union) reveals a frequent antagonism between provisioning services on the one hand, and 
regulating and cultural services on the other hand. These maps mostly account for the impacts and services provided by the main farming systems in each area. Analyzing trade-offs at a finer scale (from plot to landscape) reveals various opportunities to achieve 'win-win' situations that would preserve the environment while maintaining provisioning services. These are based on non-linear relationships between ecosystem services, grazing periods, spatial heterogeneity, system diversification, and organizational innovations between farming systems of a given area. Scenario exploration allows representing alternative dynamics for the European livestock sector in response to climate change, land use change resulting from shifts in livestock feeding, and/or a drop in animal protein consumption across Europe. We conclude by pointing out key research issues that would allow valuing the diversity of services provided by the livestock sector.

DUMONT B., RYSCHAWY J., DURU M., BENOIT M., DELABY L., DOURMAD J.Y., MÉDA B., VOLLET D., SABATIER R., 2017. Les bouquets de services, un concept clé pour raisonner l'avenir des territoires d'élevage. In : Numéro spécial, L'élevage en Europe : une diversité de services et d'impacts. Dumont B. (Éd). INRA Prod. Anim., 407-422. 
